\title{
Multi-physics numerical simulation of an experimentally predicted rubbing event in aircraft engines
}

\author{
Q. Agrapart. ${ }^{1}$, F. Nyssen ${ }^{1}$, D. Lavazec ${ }^{2}$, P. Dufrénoy ${ }^{3}$, A. Batailly ${ }^{1}$
}

\begin{abstract}
This paper provides new insight on the simulation of blade-tip/casing rubbing events within aircraft engines accounting for thermomechanical effects within the casing. A multi-physics numerical strategy is presented in order to simulate an interaction experimentally witnessed on a full-scale low-pressure compressor. Experimental data are used for an accurate representation of the blade's incursion depth within the abradable coating. This numerical strategy combines Safran's in-house tool for rotor/stator interaction simulations with a finite element based thermomechanical analysis carried out with Ansys. This work underlines the distinct contributions of both dynamical and thermomechanical phenomena in the simulated interaction. Competition between wear and thermal expansions is investigated as well as their consequences on blade dynamics. The proposed numerical strategy yields an accurate description of the interaction phenomenon as wear patterns, critical speed, amplitude growth rate of the blade vibration and temperature levels may be predicted.
\end{abstract}

Keywords

rotor-stator interaction; abradable coating; blade dynamics; thermomechanical simulation; thermal expansion

\author{
1 - Department of Mechanical Engineering, École Polytechnique de Montréal, P.O. Box 6079, Succ. Centre-Ville, Montréal, Québec, Canada H3C 3A7 \\ 2 - Safran Aircraft Engines, Villaroche, Moissy-Cramayel, 77550, France \\ 3 - Univ. Lille, CNRS, Polytech Lille, FRE 2016 - LaMcube - Laboratoire de mécanique multiphysique et multiéchelle, F-59000, Lille, France
}

\section{Stratégie numérique multi-physique pour la simulation de contacts rotor-stator dans les moteurs d'avion.}

\author{
Q. Agrapart. ${ }^{1}$, F. Nyssen ${ }^{1}$, D. Lavazec ${ }^{2}$, P. Dufrénoy ${ }^{3}$, A. Batailly ${ }^{1}$
}

\section{Résumé}

Dans cet article, la simulation de contacts entre une aube de moteur d'avion et son carter en vis-à-vis est abordée en tenant compte des effets thermomécaniques du stator. Une stratégie numérique multi-physique est présentée afin de simuler un scénario d'interaction sur la base d'un essai expérimental échelle un, réalisé sur un étage de compresseur basse pression. Les données expérimentales sont utilisées pour une représentation précise de la profondeur initiale d'incursion de l'aube dans le revêtement abradable. Cette stratégie numérique associe un outil métier Safran, pour la simulation d'interaction rotor-stator, à une simulation thermomécanique en éléments finis réalisée avec Ansys. Ce travail souligne les contributions respectives des phénomènes dynamiques et thermomécaniques dans le scénario d'interaction qui est simulé. La compétition entre usure du revêtement et dilatations thermiques du carter ainsi étudiée permet de porter un nouveau regard sur la dynamique de l'aube. La stratégie numérique apporte une description plus fine du phénomène d'interaction avec une prédiction réaliste des profils d'usure, de la vitesse critique, de l'augmentation de l'amplitude de vibration et des niveaux de température.

\section{Mots-clés}

interaction rotor-stator; revêtement abradable; dynamique d'aube; simulation thermomécanique; dilatations thermiques

1 - Département de génie mécanique, École Polytechnique de Montréal, P.O. Box 6079, Succ. Centre-Ville, Montréal, Québec, Canada H3C 3A7 2 - Safran Aircraft Engines, site de Villaroche, Moissy-Cramayel, 77550, France

3 - Univ. Lille, CNRS, Polytech Lille, FRE 2016 - LaMcube - Laboratoire de mécanique multiphysique et multiéchelle, F-59000, Lille, France 


\section{Introduction}

The understanding of blade-tip/casing interactions in general and rubbing events in particular is a priority for aircraft engine manufacturers and blade designers. Indeed, the drive towards tighter operating clearances, which yields higher engine efficiency, unavoidably promotes these undesired events. The intricacy of the physical phenomena at play is due to a combination of: (1) sophisticated rotordynamics [1, 2], (2) high speed structural contact events [3], (3) complex wear mechanisms within the abradable sealing [4, 5] and (4) aerodynamic forcing over the blades [6]. Commercial software packages are not suited for an efficient analysis of these interactions: it is not currently possible to combine accurate contact treatment, efficient rotor modeling and wear mechanisms while keeping acceptable computation times. For this reason, manufacturers rely on ad-hoc numerical strategies $[7,8]$ that must be thoroughly confronted to experimental data in order to ensure robust numerical predictions.

In this context, research laboratories and engine manufacturers collaborate for the development of predictive numerical tools: experimental set-ups designed by or with the manufacturers provide research labs the much needed data to calibrate these tools. There exists a significant number of experimental facilities dedicated to the analysis of blade-tip/casing interactions $[9,10,11,12,13,14,15,16,11,17]$, some of them focusing on the interaction phenomenon itself [11,14, 15], or on full-scale configurations with the intent to reproduce engine-like conditions [11, 17]. Material characterizations have been carried out on different types of abradable coatings $[18,13,19]$ that may be found within turbo-engines. Their classification mainly depends on the operating temperature: (1) metallic coatings with polymer matrix and solid lubricant are used for moderate temperatures $\left(<500^{\circ} \mathrm{C}\right)$ in compressor stages, $(2)$ porous sintered metal materials, honey comb structures, or ceramic coatings are needed under more severe conditions $\left(<1300^{\circ} \mathrm{C}\right)$ in turbine stages. While initially driven by purely structural aspects [20, 11], research on blade-tip/casing interactions early focused on abradable coating removal modeling. As a matter of fact, specific wear patterns observed post-mortem on the casing suggest that wear played a key role in divergent interactions [21, 22]. The aforementioned interactions have also been witnessed under vacuum [23], underlining the intrinsically structural nature of the interaction which is thus not related to aerodynamic sollicitations. For this reason, aerodynamic loadings are usually not accounted for in numerical strategies focusing on the prediction of blade-tip/casing interactions.

More recently, experimental observations made on several full-scale experimental set-ups underlined very significant temperature increases in the vicinity of contact areas as well as within the whole stator [21, 23, 24]. These temperature increases are such that they may alter the abradable coating mechanical properties and yield significant thermal expansions on both the casing and the impacting blades. Accordingly, most recent research efforts have focused on developing efficient thermomechanical modeling strategies. For instance, in the context of helicopter engines featuring smaller and very stiff casings, thermomechanical reduced order models have recently been developed [25]. Also, within the high-pressure compressor of aircraft engines, numerical models have been proposed for the prediction of temperature increases within the abradable sealing [26]. Yet, so far, there exists no numerical strategy able to accurately predict and account for localized thermal expansions on the casing surface.

For this reason, the influence of thermal effects on the casing itself has not yet been investigated and certain experimentally observed interactions, such as the first contact configuration described in [23], have never been successfully numerically simulated accounting for realistic levels of interpenetration between the blade and the abradable coating.

The specificity of the first contact configuration presented in [23] lies in the fact that it is estimated the blade only rubs away a few microns of abradable coating per revolution. For such contact configuration, existing numerical strategies predict at best localized wear but no significant amplitude of vibration is anticipated for the blade. This goes against experimental observations where blade failure was evidenced after about a hundred seconds.

Based on the numerical strategy previously presented in [24], and building on recent numerical developments [27], this article introduces a multi-physics numerical strategy dedicated to the prediction of blade-tip/casing interactions within aircraft engines accounting for wear and thermomechanical effects on the casing. An existing numerical strategy dedicated to the vibration analysis of an industrial aircraft engine blade accounting for abradable coating wear is coupled with a thermomechanical procedure allowing to compute localized thermal expansions on a full 3D finite element model of an aircraft engine casing. The focus is made on preserving acceptable computation times while ensuring a high resolution of the investigated thermomechanical problem. As a consequence, particular attention is paid to the convergence of the proposed numerical strategy, with respect to both time and space parameters. 
The second section of the article recalls the experimental set-up on which has been witnessed the interaction used for calibration of the proposed numerical strategy. In the third section, the strongly coupled thermomechanical methodology is detailed, before its convergence be thoroughly assessed with respect to all numerical parameters in the fourth section. Finally, in the fifth section, the proposed numerical strategy is confronted to experimental results: the quality of the results is assessed in terms of critical speed, wear patterns on the abradable sealing, outer casing temperatures and blade dynamics.

\section{Experimental set-up}

Details about the experimental set-up may be found in [23]. Key aspects related to the proposed developments are recalled here for the sake of completeness. Two distinct contact configurations are presented in [23]. The focus is made on the first interaction (7-lobe interaction, in reference to the number of wear lobes observed along the casing contact surface after the interaction). The second configuration (6-lobe interaction), featuring a more severe contact configuration with a greater penetration depth of the blade within the abradable coating, has previously been numerically investigated in [22] without accounting for thermomechanics.

\subsection{Test rig}

\subsubsection{Engine components}

The experimental set-up is based on a full scale low-pressure compressor stage of a civil aircraft engine. A blade mounted disk and its surrounding casing are isolated to be assembled on the test bench, see Fig. 1. During the run test, centrifugal effects yield closure of the blade/casing clearance so that contact is established between the two structures. It is possible to know a priori which blade will be involved in the interaction since one blade is slightly longer than the other ones. A dynamic balancing is performed in order to allow such a contact configuration. The effect of mistuning is assumed to be negligible, which is experimentally confirmed as additional strain gages on both the disk and neighbouring blades highlight that amplitudes of vibration are only significant on the longer blade. Also, the inner surface of the casing is thermally sprayed with an abradable coating made from an AlSi-Po composite powder. This yields a metal base matrix embedded in a polymer lattice, with about $60 \% \mathrm{Al}-\mathrm{Si}$ and $40 \%$ Po in volume. The manufacturing process of the AlSi-Po abradable coating also generates porosity which ratio is less than $5 \%$ in volume. This coating was chosen for its abradability - which characterizes how easily it may be worn off - and its resistance to erosion in a turbo-engine environment. This family of abradable materials can be found in low and high pressure compressor stages, with moderate operating temperatures $<500^{\circ} \mathrm{C}$. However, engine-like conditions are not intended to be reproduced because run tests are performed under vacuum at room temperature.

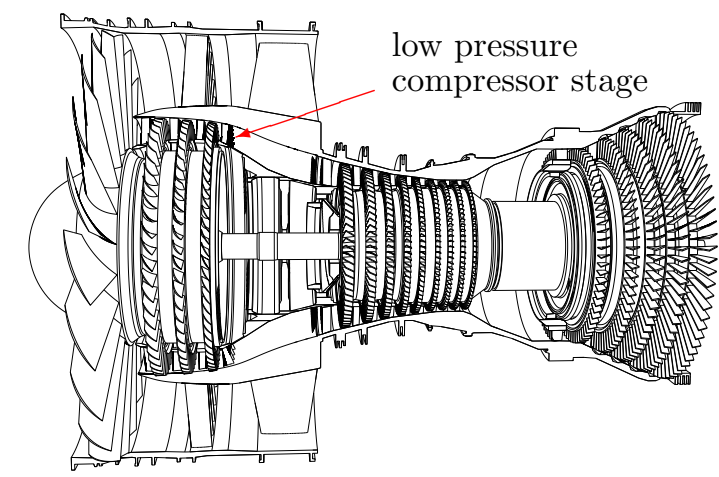

(a) schematic cut view of a turboengine

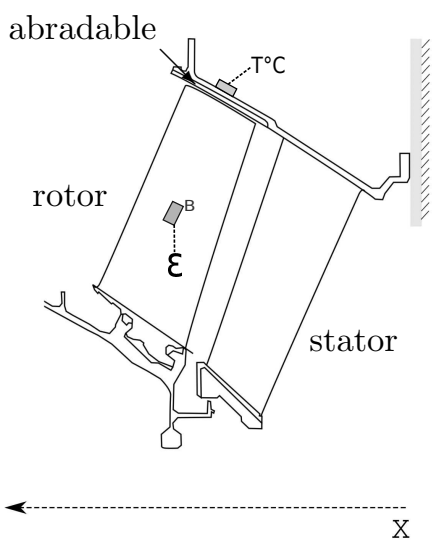

(b) rotor geometry and its surrounding casing covered with an abradable coating

Figure 1. geometry of the low-pressure compressor stage used in the experimental set-up 
Partial profile measurements - measured at only four points along the circumferential direction - on the casing inner surface reveal a maximum radial deformation of about $0.45 \mathrm{~mm}$, to be compared to the $1.5 \mathrm{~m}$ inner diameter of the casing. Minimum casing radii are localized at $\theta=\pi / 2$ and $\theta=3 \pi / 2$ in the polar plots displayed in Figure 5 . Accordingly, it is expected that contact will be initiated at one of these two areas. There is no other available data to assess more precisely the overall shape of the casing. However, these measurements highlight an ovalization of the inner profile, that was expected due to specific mounting conditions.

\subsubsection{Targeted angular speed}

The evolution of the blade's three first eigenfrequencies - corresponding to first bending (1B), second bending (2B) and first torsional (1T) modes - with respect to the angular speed is given in the Campbell diagram plotted in Fig. 2. The targeted angular speed for contact interaction is denoted $\Omega_{\mathrm{h} 7}$ which is located in the vicinity of the intersection of mode $1 \mathrm{~B}$ with the seventh engine order $\left(e_{o}=7\right)$.

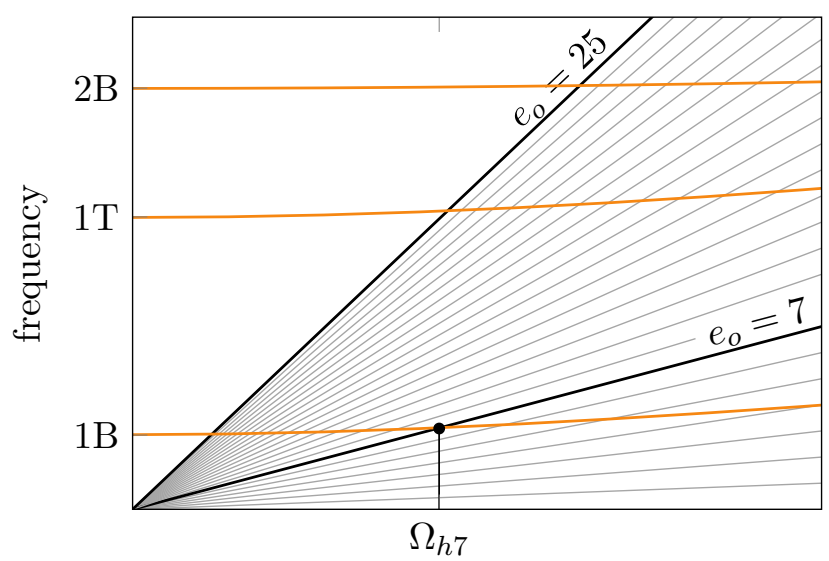

$\Omega$

Figure 2. Campbell diagram

It is experimentally observed that the targeted speed is not high enough to initiate contact. It is found that the angular speed $\Omega_{h 7}$ must be increased by $1 \%$ in order to detect the first contact. The angular speed then remains constant at $\Omega=1.01 \Omega_{h 7}$ throughout the interaction. Contrary to the second configuration mentioned in [23], the very small increase of angular speed that leads to contact initiation indicates a very soft contact scenario: it is estimated that the blade only removes a few microns of abradable coating during the first revolutions following contact initiation.

\subsection{Measurements and observations}

The aforementioned contact configuration yields blade failure within a hundred seconds following the first contact. Post-mortem analyses from [23] indicate the presence of potentially harmful mechanisms: increasing amplitudes of vibration, significant wear levels in the abradable coating, significant temperature increases as well as cracks in the vicinity of the blade root.

\subsubsection{Dynamic response of the blade}

The time response of one of the instrumented blade's strain gauges (gauge B, see Fig. 1) is given in Fig. 3. The origin of this signal $(t=0 \mathrm{~s})$ is the first blade/casing contact. Three distinct phases were identified in [23] :

phase 1: $t \in[0,60] \mathrm{s}$, a small but continuous increase of amplitudes is evidenced over the first minute of the interaction,

phase 2: $t \in[60,107] \mathrm{s}$, amplitudes of vibration stabilize around a high value for about 40 seconds,

phase 3: $t \in[107,116] \mathrm{s}$, a sudden burst of amplitudes is witnessed during a short amount of time, it leads to blade failure. 


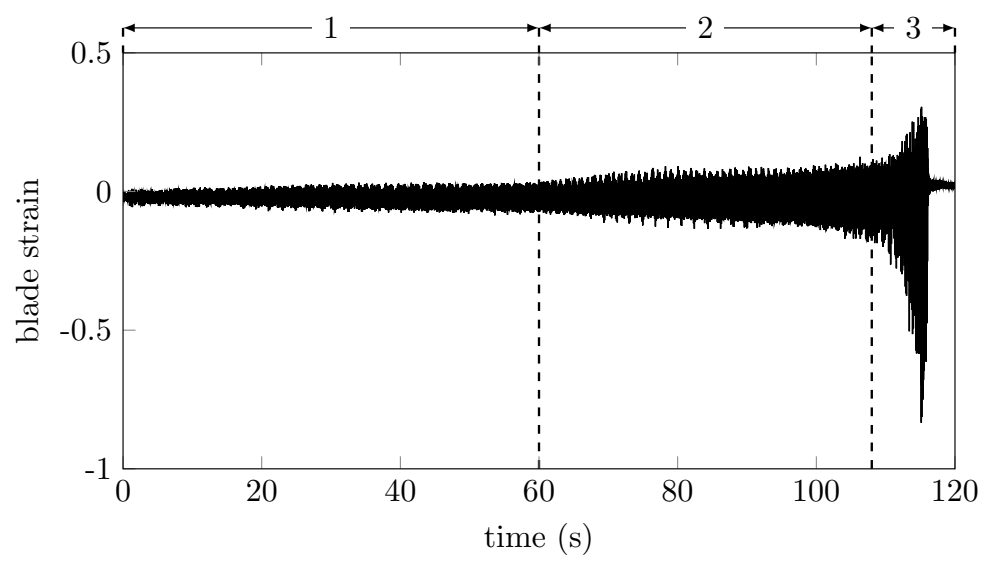

Figure 3. normalized blade strain (gauge B) adapted from [23]

A short-time Fourier transform of the signal plotted in Fig. 3 yields the spectrogram depicted in Fig. 4a where amplitudes are discriminated using a color code from white - for lowest amplitudes - to black - for highest amplitudes. For the considered angular speed, there is a coincidence between the first bending mode and engine order $e_{\mathrm{o}}$ as well as the first torsional mode and engine order $e_{\mathrm{o} 25}$. As a consequence, highest amplitudes are found around these two engine orders. Previously identified phases 1,2 and 3 each feature a distinctive frequency spectrum.

At the beginning of the interaction, highest amplitudes of vibration are located around $e_{\mathrm{o} 25}$ which indicates a dominant contribution of mode $1 \mathrm{~T}$ in the blade's response. Meanwhile, all along phase 1, the amplitude of vibration along $e_{\mathrm{o} 7}$ increases steadily. As the contribution of the first bending mode increases, the transition between phases 1 and 2 is characterized by a drop of amplitude along $e_{\mathrm{o} 25}$, see Fig. $4 \mathrm{~d}$. During phase 2, the contribution of the first bending mode increases significantly as the one of the first torsional mode decreases to a point where it becomes negligible. Once the contribution of the first torsional mode has vanished, the blade's dynamics is solely related to the first bending mode: a burst of amplitude is evidenced in phase 3 .

\subsubsection{Wear pattern on the abradable coating}

Measurements of wear profiles are realized at three axial positions: in front of the leading edge, the middle of the chord and the trailing edge. Post-mortem wear levels are plotted in Fig. 5 using a polar representation. The final wear profile at the leading edge is noticeably different from the one observed at other axial positions: seven deep wear lobes are evidenced. Locally, the abradable coating has been fully worn off making it possible to see the casing titanium material. Post-mortem experimental observations are most likely representative of the blade dynamics during its last revolutions as the history of wear patterns are overlayed during the successive contact events.

\subsubsection{Temperature probes}

Several temperature probes are located on the outer face of the casing facing the middle of the chord as shown in Fig. 1. Thermal data are monitored at different angular locations highlighted in Fig. 5 and are superimposed to the wear profile at the middle of the chord. Temperature rises at the end of phase 3 are displayed on their respective sensors, see Fig. 5b. Temperatures on the back of the casing range from $+15^{\circ} \mathrm{C}$ to $+125^{\circ} \mathrm{C}$. The limited number of probes does not allow to make any correlation between temperature increases and wear levels. Also, there was no temperature probe on the blade.

\subsection{Summary of the experiment}

The interaction of interest is characterized by a very low penetration depth of the blade within the abradable coating and blade failure occurs more than one hundred seconds following the first contact. Three distinct phases are identified but partial thermal data do not allow to associate temperature increases with any of these phases. Because temperatures are measured on the outer surface of the casing, one may expect extremely high temperatures 


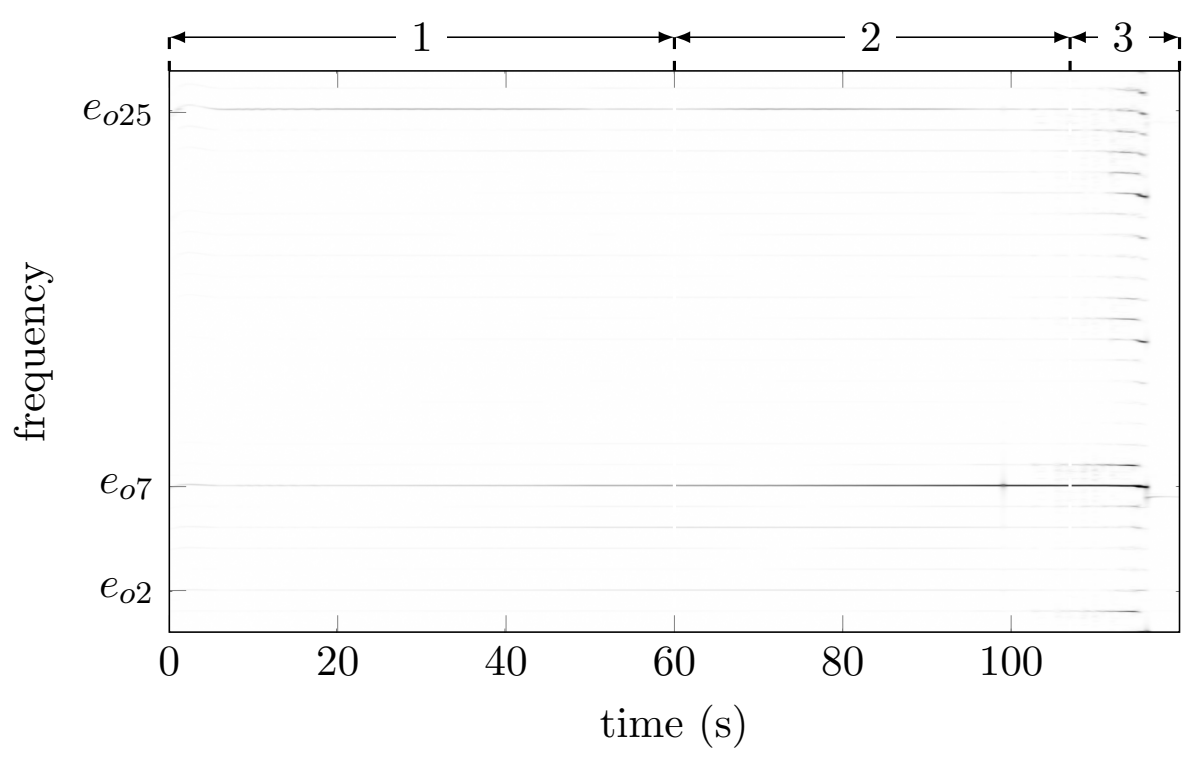

(a) time-frequency representation

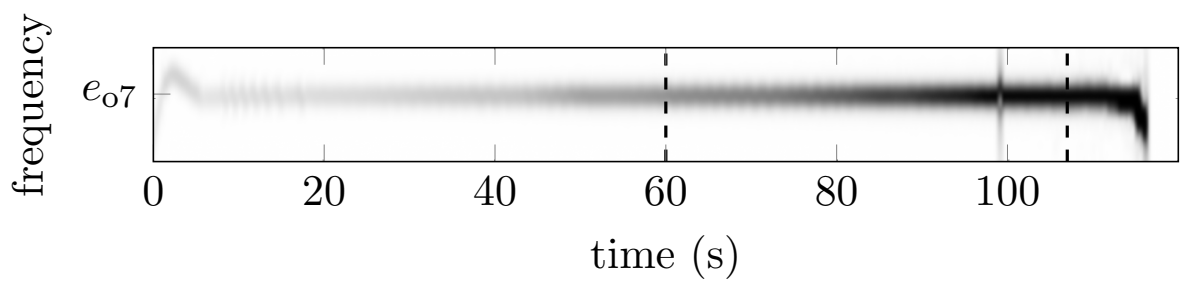

(b) focus on $e_{07}$

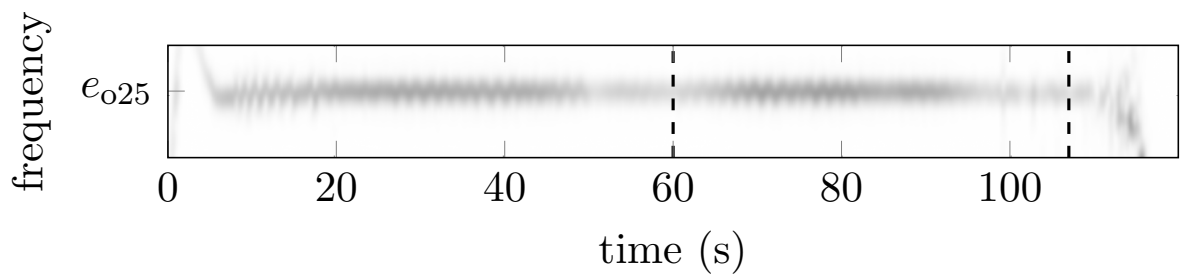

(c) focus on $e_{o 25}$

$$
0
$$

(d) colorbar

Figure 4. spectrogram of gauge B strain signal

along the casing contact surface thus implying non negligible thermal expansions [21, 27] that may significantly influence the blade/casing contact configuration. Thus, it is assumed that an accurate numerical prediction of the witnessed interaction calls for a strongly coupled thermomechanical strategy.

All experimental observations are certainly highly dependent on the type of abradable coating, geometries, and test parameters. This paper does not intend to present a solution to the arisal of the observed interaction which can occur in a turbo-engine. Instead, the focus is made on the simulation of this given interaction. 


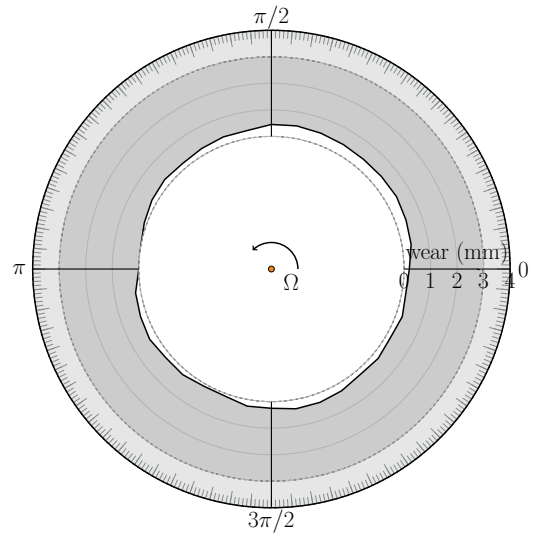

(a) leading edge

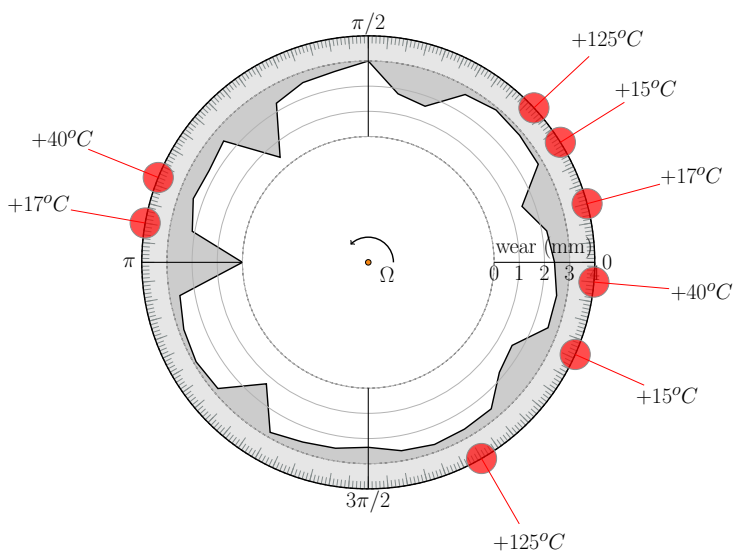

(b) middle of chord

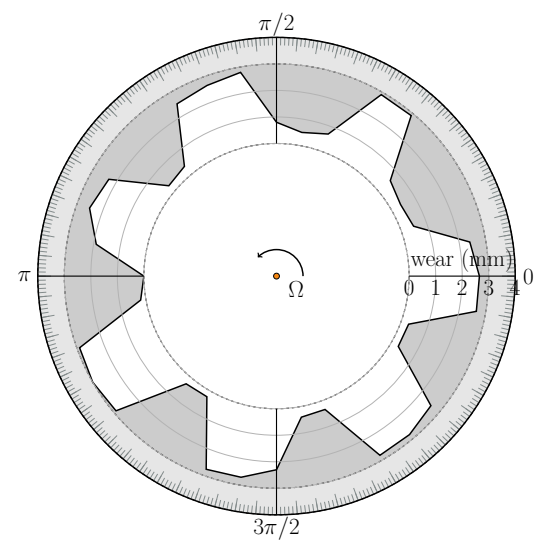

(c) trailing edge

Figure 5. circumferential wear profiles adapted from [23]

\section{Multi-physics numerical strategy}

The proposed developments focus on the addition of thermomechanical considerations to the numerical strategy previously presented in [8], hereafter referred to as COROS (COntact ROtor Stator). The resulting multi-physics numerical strategy allows to simulate rubbing events accounting for contact, abradable coating wear and thermal expansion of the casing. The description of the numerical framework related to contact treatment and wear may be found in $[8,28]$, it is only briefly recalled in section 3.1 for the sake of completeness. To the contrary, developments related to the modeling of thermomechanics - which are original - are presented in details. The diagram in Fig. 6 provides a detailed view of the new algorithm alongside previously published developments.

\subsection{Contact and wear}

The contact algorithm allowing wear management is shown in the COROS blue box in Fig. 6. An accurate representation of the rotor's dynamics - be it a blade or a bladed disk - is achieved by means of component mode synthesis techniques applied on industrial full 3D finite element models [8]. Inertial effects (centrifugal and gyroscopic) may be accounted for on the resulting reduced order model. Because most of the experimentally observed interactions are inherently unsteady (see, for instance, the time response in Fig. 3), contact interactions are numerically simulated using time integration. An explicit central finite differences time integration scheme [29] is here employed. Contact treatment along the blade chord is ensured by means of a Lagrange-multiplier based algorithm [29] acting on a few 
physical degrees of freedom kept within the rotor's reduced order model. Abradable coating wear is computed by means of a plastic constitutive law [28] providing a live update of blade/casing clearances as contacts occur. Also, and in agreement with experimental observations, the casing is typically modeled as a perfectly rigid - potentially deformed - structure: it does not vibrate due to contact. This numerical strategy, COROS, has previously been validated with respect to several experimental results obtained on both full-scale experimental set-ups [22, 24] as well as simplified test benches [30].

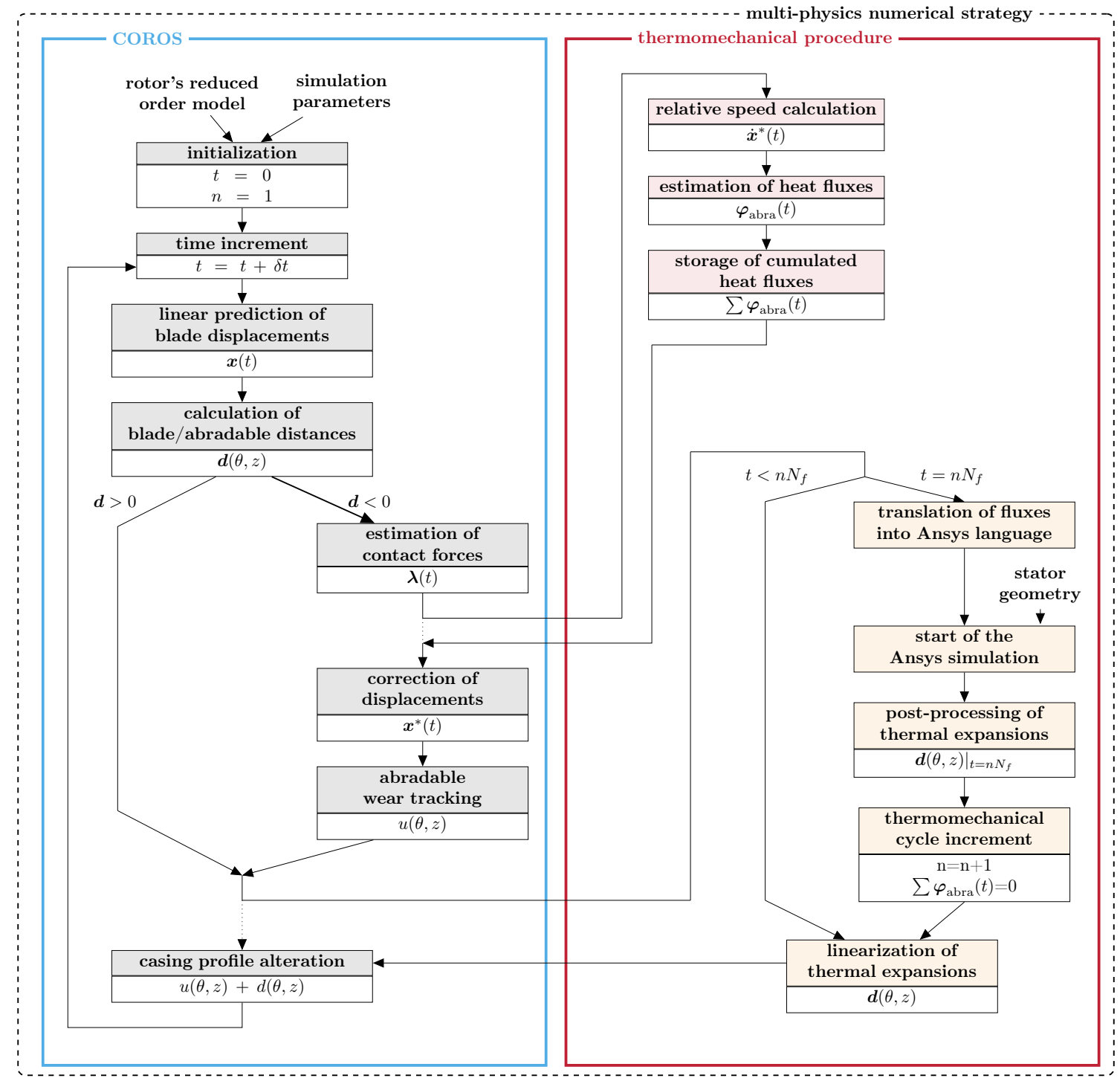

Figure 6. diagram of the multi-physics numerical strategy which couples COROS and the thermomechanical procedure (ANSYS)

\subsection{Thermomechanics}

Very high temperatures in the vicinity of impacted areas along the casing surface have been reported in several published experimental investigations [23, 24]. The resulting thermal load on the casing may have a twofold effect: (1) locally, as impacted areas are heated, thermal expansions may reduce blade/casing clearances and (2) globally, the heating of the casing may yield its distortion and thus modify blade/casing clearances at any angular location 
depending on the casing mounting points. Thermomechanics on the rotor [31, 32] is not considered in this study as there is no experimental evidence that very localized heated areas - along the blade tip - may significantly impact its dynamics.

\subsubsection{Coupling strategy}

Original developments related to the thermomechanical procedure are underlined in red in Fig. 6 . The key elements of the methodology are detailed below and illustrated by means of the experimental casing. In a more general context, the multiphysics numerical strategy may easily be adjusted to any stator geometry and any thermal or mechanical boundary conditions to reproduce engine-like conditions. The proposed approach relies on the fact that blade dynamics and casing thermomechanics feature very distinct time scales. Indeed, while the simulation of contact events requires an explicit time integration procedure with very small time steps (typically $\delta t=10^{-7} \mathrm{~s}$ ), thermomechanics may be solved using time steps six or seven orders of magnitude larger (up to $\delta t_{\text {th }}=1 \mathrm{~s}$ ).

A schematic representation of the proposed coupled numerical strategy is depicted in Fig. 7. Resolution of the

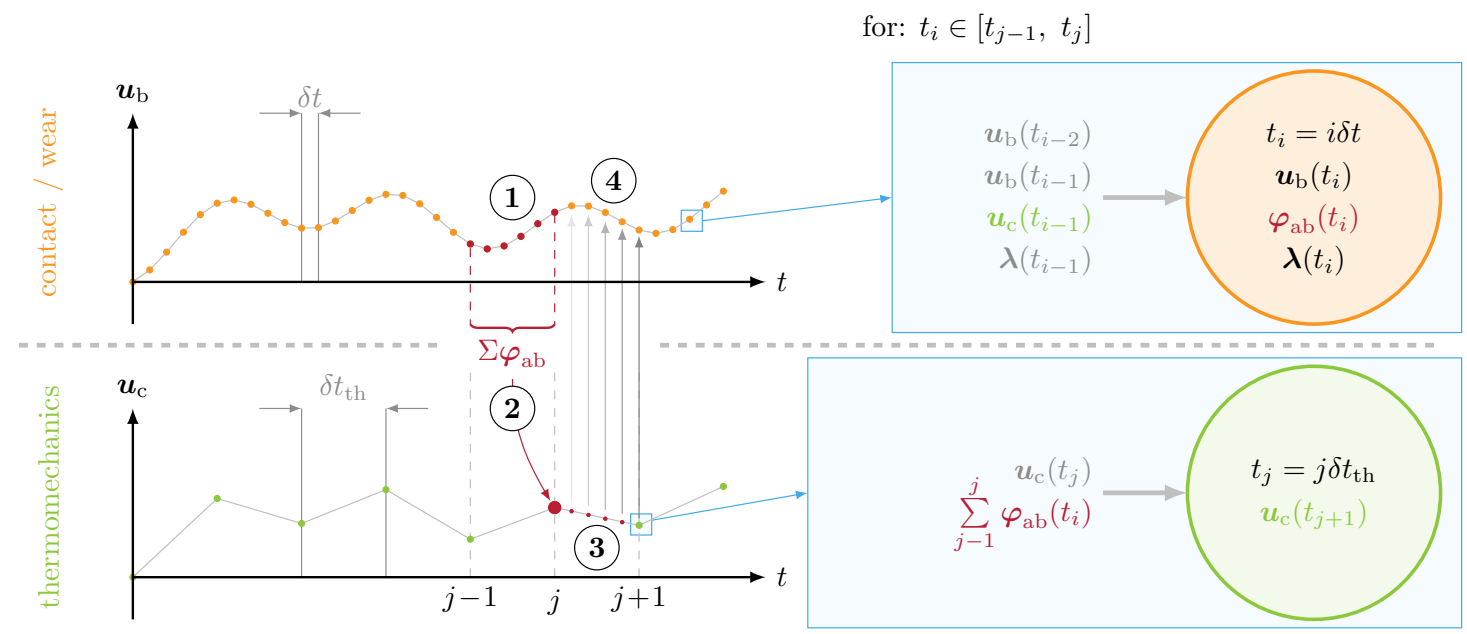

Figure 7. temporal representation of the global strategy

dynamic problem by COROS - that includes contact, blade vibrations and abradable coating wear - is numerically alternated with thermomechanical computations on the casing using the Ansys 16.2 commercial software.

In the following, $i$ refers to the $i^{\text {th }}$ step of the dynamic problem, and $j$ to the $j^{\text {th }}$ step in the thermomechanical problem. Accordingly, notations $t_{i}=i \cdot \delta t$ and $t_{j}=j \cdot \delta t_{\text {th }}$ are used to refer to a specific instant of each computation. Key steps of the methodology are:

1. COROS is run for $t_{i} \in\left[t_{j-1}, t_{j}\right]$ in order to compute blade displacement vector $\boldsymbol{u}_{\mathrm{b}}\left(t_{i}\right)$, contact forces vector $\boldsymbol{\lambda}\left(t_{i}\right)$ and dissipated heat flux vector $\boldsymbol{\varphi}_{\mathrm{ab}}\left(t_{i}\right)$,

2. the heat flux transferred to the casing $\sum_{j-1}^{j} \varphi_{\mathrm{ab}}\left(t_{i}\right)$ due to friction over $t_{i} \in\left[t_{j-1}, t_{j}\right]$ is computed (see section 3.2.2),

3. $\sum_{j-1}^{j} \boldsymbol{\varphi}_{\mathrm{ab}}\left(t_{i}\right)$ is the input of a two-step thermomechanical analysis carried out with the Ansys commercial software which provides as output the thermal expansions on the casing $\boldsymbol{u}_{\mathrm{c}}\left(t_{j+1}\right)$ :

- transient thermal analysis, see section 3.2.3,

- static thermomechanical analysis, see section 3.2.4,

4. COROS is run over $t_{i} \in\left[t_{j}, t_{j+1}\right]$ considering the casing thermal expansions $\boldsymbol{u}_{\mathrm{c}}\left(t_{j+1}\right)$ which is linearly applied over time 
5. steps 2 to 4 are then repeated until the end simulation time has been reached.

\subsubsection{Heat flux transfered to the casing $\varphi_{\mathrm{ab}}\left(t_{i}\right)$}

The computation of heat fluxes $\boldsymbol{\varphi}_{\mathrm{ab}}\left(t_{i}\right)$ is related to the work of interaction forces $\delta \boldsymbol{W}\left(t_{i}\right)$-which represents the amount of energy dissipated at the interface during a contact event-, see Eq. (1). There are two distinct terms in Eq.(1): one related to the contribution of interaction forces $\boldsymbol{\lambda}^{n}\left(t_{i}\right)$ in the normal direction, and one related to the work done by tangential forces $\boldsymbol{\lambda}^{t}\left(t_{i}\right)$. Superscripts ${ }^{n}$ and ${ }^{t}$ respectively indicate the restriction of a vector to its coordinates along the normal and tangential directions.

$$
\delta \boldsymbol{W}\left(t_{i}\right)=\boldsymbol{\lambda}^{n}\left(t_{i}\right)\left[\boldsymbol{u}_{\mathrm{b}}^{n}\left(t_{i}\right)-\boldsymbol{u}_{\mathrm{b}}^{n}\left(t_{i-1}\right)\right]+\boldsymbol{\lambda}^{t}\left(t_{i}\right)\left[\boldsymbol{u}_{\mathrm{b}}^{t}\left(t_{i}\right)-\boldsymbol{u}_{\mathrm{b}}^{t}\left(t_{i-1}\right)\right] \quad[J]
$$

The associated instantaneous power $\boldsymbol{P}\left(t_{i}\right)$ is given in Eq. (2). It is assumed that the contribution of the work related to the normal direction is negligible in comparison to the work related to the tangential directions. Indeed, the order of magnitude of the rubbing distance increment $\boldsymbol{u}_{\mathrm{b}}^{t}\left(t_{i}\right)-\boldsymbol{u}_{\mathrm{b}}^{t}\left(t_{i-1}\right)$ is far greater than the variation of the depth of incursion $\boldsymbol{u}_{\mathrm{b}}^{n}\left(t_{i}\right)-\boldsymbol{u}_{\mathrm{b}}^{n}\left(t_{i-1}\right)$ due to the rotation of the blade. Because the radial contribution has been neglected, $\boldsymbol{P}\left(t_{i}\right)$ also represents the power dissipated by friction:

$$
\boldsymbol{P}\left(t_{i}\right)=\boldsymbol{\lambda}^{t}\left(t_{i}\right) \frac{\boldsymbol{u}_{\mathrm{b}}^{t}\left(t_{i}\right)-\boldsymbol{u}_{\mathrm{b}}^{t}\left(t_{i-1}\right)}{\delta t}=\mu \boldsymbol{\lambda}^{n}\left(t_{i}\right) \dot{\boldsymbol{u}}_{\mathrm{b}}^{t}\left(t_{i}\right) \quad[W]
$$

A standard Coulomb model is used, the friction coefficient is denoted $\mu$ so that $\boldsymbol{\lambda}^{t}\left(t_{i}\right)=\mu \boldsymbol{\lambda}^{n}\left(t_{i}\right)$. The time derivative of displacements $\dot{\boldsymbol{u}}_{\mathrm{b}}^{t}\left(t_{i}\right)$ represents the relative speed that includes both the rotor revolution and the self vibratory motion of the blade collinear over the sliding direction.

Due to unavoidable losses and a variety of thermomechanical phenomena, the power that actually contributes to heating the casing is computed in COROS as:

$$
\boldsymbol{\Phi}_{\mathrm{ab}}\left(t_{i}\right)=p_{\mathrm{ab}} \mu \boldsymbol{\lambda}^{n}\left(t_{i}\right) \dot{\boldsymbol{u}}_{\mathrm{b}}^{t}\left(t_{i}\right), \text { with: } 0<p_{\mathrm{ab}}<1
$$

Studies on energy balances conducted on experimental test rigs [27, 33] have shown that parameter $p_{\text {ab }}$ depends on incursion parameters, material properties and activated wear mechanisms. It is here assumed that $p_{\mathrm{ab}}=0.9$, based on empirical data and recent numerical investigations [27]. The same process may be used for investigating different interaction configurations but dedicated analyses may be required to accurately estimate the value of $p_{\text {ab }}$. The execution of COROS over a given number of time steps thus provides data associated to impacted areas and their local heat flux. Figure 8 illustrates typical predicted contact areas over a certain number of time steps.

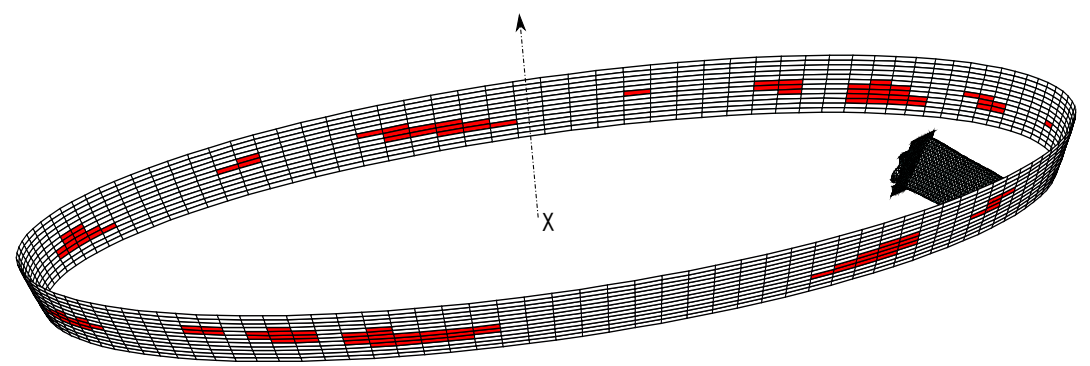

Figure 8. blade and casing inner contact surface, impacted areas over a revolution are marked in red

Heatings in the Ansys thermal simulation are then introduced by applying heat flux $\varphi_{\mathrm{ab}}\left(t_{i}\right)$-an energy per unit of area per unit of time - on the surface $s_{e}$ of impacted elements:

$$
\varphi_{\mathrm{ab}}\left(t_{i}\right)=\boldsymbol{\Phi}_{\mathrm{ab}}\left(t_{i}\right) / s_{\mathrm{e}}
$$

which allows to carry out the transient thermal analysis. 


\subsubsection{Thermal analysis}

Transient thermal analyses are performed using a 3D finite element model of the casing with the Ansys commercial software. The procedure is based on an uncoupled approach to solve thermal and mechanical problems sequentially.

The input of the thermal analysis is the sum of all the heat fluxes $\varphi_{\mathrm{ab}}\left(t_{i}\right)$ computed with COROS for $t_{i} \in\left[t_{j-1}, t_{j}\right]$, denoted $\sum_{j-1}^{j} \varphi_{\mathrm{ab}}\left(t_{i}\right)$. COROS provides a very high resolution of heat fluxes with respect to time due to the very small time integration step used for contact management $\delta t$. An exact representation of these fluxes in Ansys requires the transient thermal analysis time step be equal to $\delta t$ thus yielding extremely long computation times. In order to maximize the efficacy of the thermal analysis, the sum of heat fluxes on a given element for $t_{i} \in\left[t_{j-1}, t_{j}\right]$ is applied over a period of time $\delta t$, see Fig. 9. By doing so, only two steps are required for the transient thermal analysis which significantly reduces computation times. The convergence analysis carried out in the next section of the paper justifies a posteriori this choice.

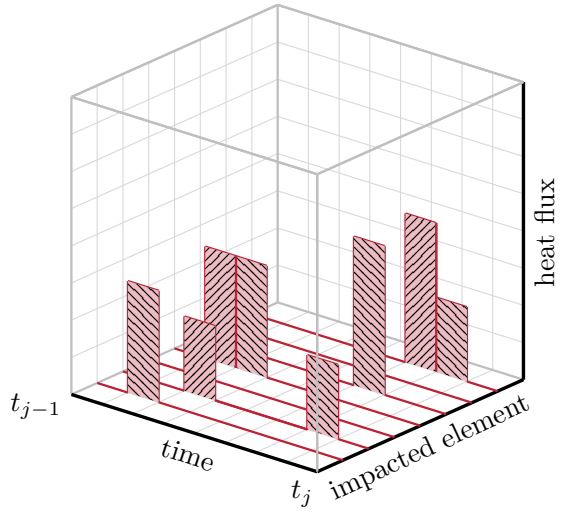

(a) $\Sigma \varphi_{a b}$ predicted by COROS

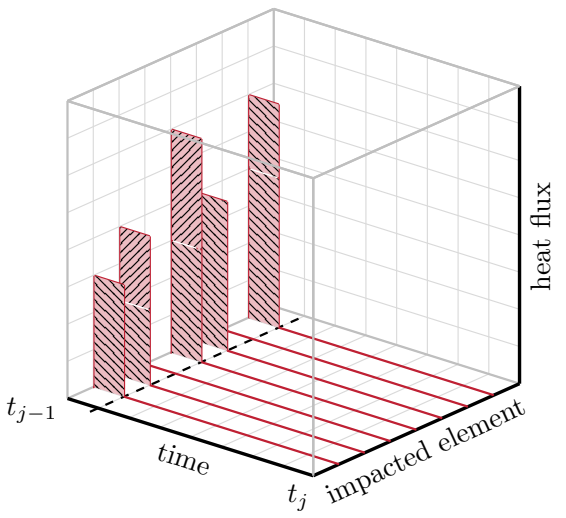

(b) $\Sigma \varphi_{a b}$ used in Ansys

Figure 9. time scenario of the transient thermal analysis

In the context of high speed rubbing, the thermal diffusion in the $\theta z$-plane becomes negligible when the relative speed between the blade and the casing is fairly high [34]. The Peclet number $P e$ is a usefull dimensionless indicator to simplify computational models in certain situations. Indeed, high values $P e>5$ [34] ensure that thermal phenomena are directed exclusively in the direction normal to the rubbed surface, orthogonal to the sliding direction. The calculation of $P e$ is given in Eq. (5) and involves the sliding speed, the contact geometry and the thermal properties of the rubbed body:

$$
P e=\frac{\dot{u}_{b}^{t} \cdot d}{\alpha}
$$

with $\dot{u}_{b}^{t}$ the relative speed over the sliding direction, $d$ the characteristic length of the carrying contacting surface and $\alpha$ the thermal diffusivity of the abradable coating.

The value of $P e$ in this study reaches $P e=70,000$ which indicates that thermal phenomena are essentially related to the radial direction.

The full 3D finite element model of the casing is represented in Fig. 10. The investigated stator is a one-piece structure on which is sprayed the abradable coating. The experimentally measured ovalization of the inner surface is accounted for. The inner contact surface on which the blade will rub is delimited by two red lines. Initially, for $t=0 \mathrm{~s}$, the casing is supposed to have a uniform temperature $T_{0}=20^{\circ} \mathrm{C}$. In the end, the thermal analysis provides the temperature field within the casing at $t_{j+1}$. 


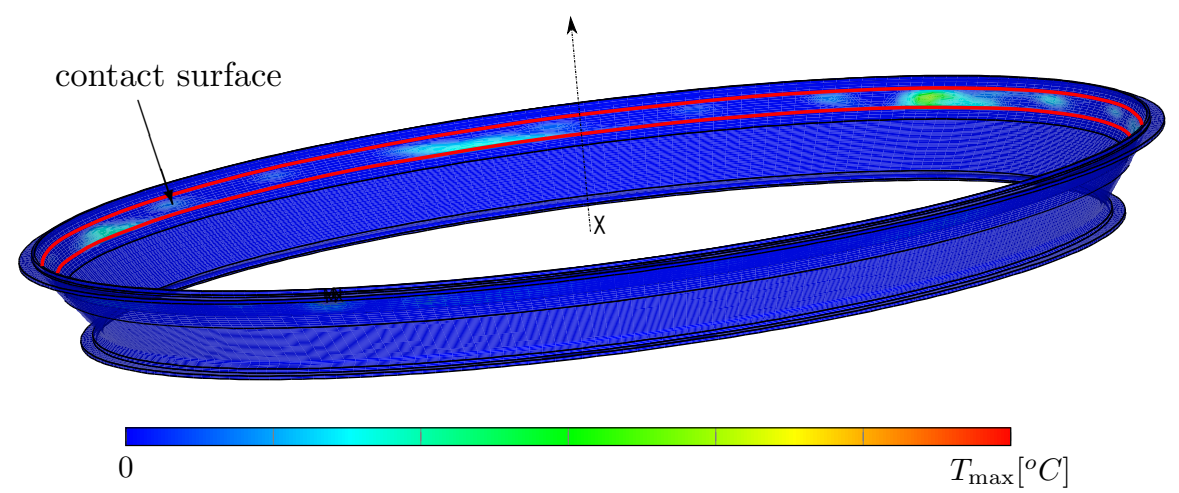

Figure 10. full finite element model of the casing with increased temperatures on the contact surface

\subsubsection{Thermomechanical analysis}

The temperature field within the casing yields thermal strains and a distortion of the inner contact surface thus impacting the blade/casing contact configuration. Applying the temperature field at $t_{j+1}$ computed from the thermal analysis as boundary conditions on the casing, the static thermomechanical analysis provides the resulting displacement field on the casing $\boldsymbol{u}_{\mathrm{c}}\left(t_{j+1}\right)$. Coupling between thermal and mechanical aspects is reflected in the thermo-elasticity properties defined for abradable and titanium casing materials.

Boundary conditions in terms of displacements applied on the casing are highlighted in Fig. 11. Clamped areas, marked in red, are consistent with the mounting configuration of the casing in the experimental set-up.

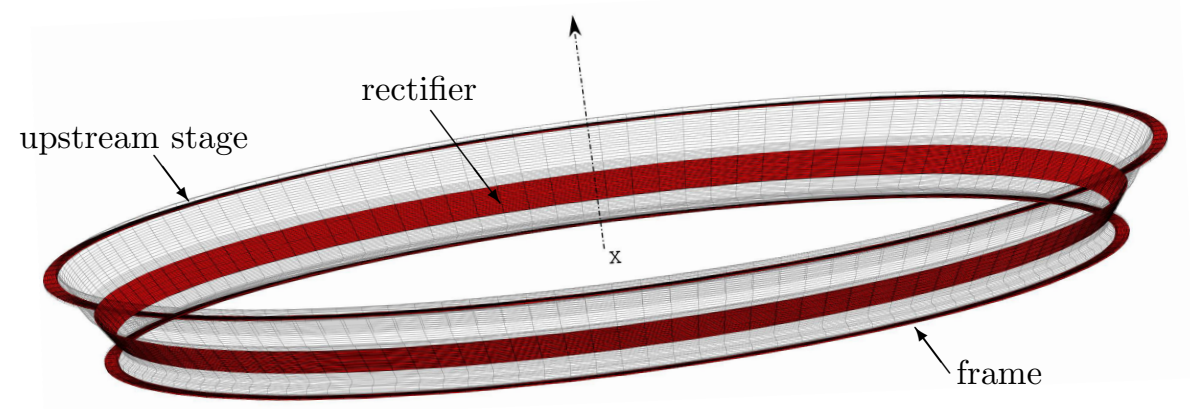

Figure 11. boundary conditions on the casing, clamped areas are marked in red

An example of displacements induced by thermal effects is given in Fig. 12. The color map displays the total displacement level obtained by considering the thermal load proposed in Fig. 10. The display scale has been widely increased by a factor 1000 to better visualize localized thermal expansions. Thermomechanical finite element simulations show that the gap is closed when heat is dissipated in the contact surface, regardless of the loadings uniformity. This is due to the stator geometry and its boundary conditions, which lead to an axial distortion of the casing. It can also be noted that the abradable coating serves as a thermal barrier with a low conductivity given by its polymer phase. At first, thermal expansions are localized around the contact area and the gap is closed in a very localized way in dependence upon the time scale.

\subsubsection{Linearization of thermal expansions}

The displacement field of the casing $\boldsymbol{u}_{\mathrm{c}}\left(t_{j+1}\right)$ is the final result of the thermomechanical procedure illustrated in Fig. 7. This displacement field must then be sent back into the COROS numerical procedure: it is smoothly 


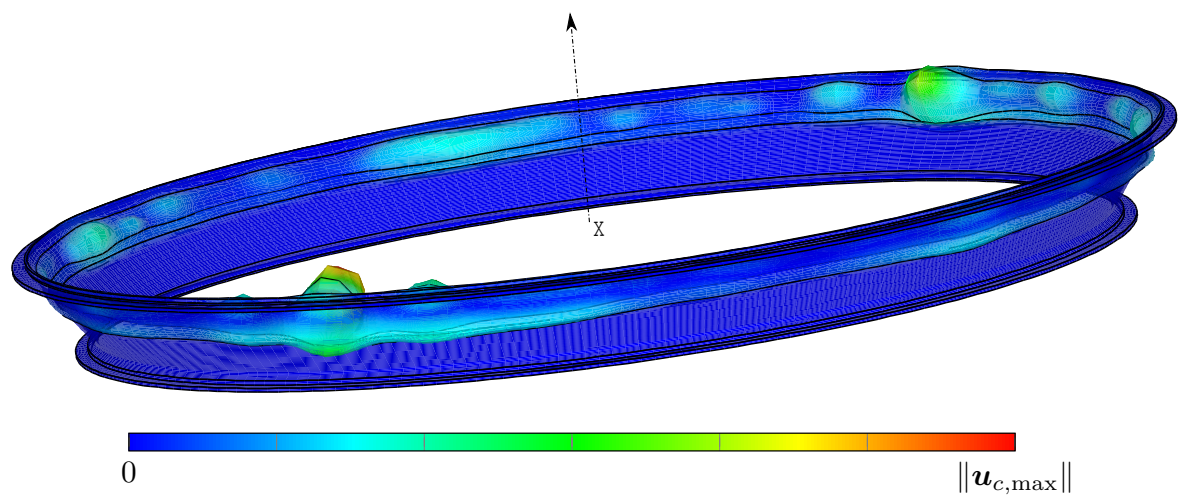

Figure 12. example of displacement field computed with a thermomechanical analysis (magnification factor: 1,000)

accounted for over the time interval $\left[t_{j}, t_{j+1}\right]$ considering for each $t_{i} \in\left[t_{j}, t_{j+1}\right]$ :

$$
\boldsymbol{u}_{\mathrm{c}}\left(t_{i}\right)=\boldsymbol{u}_{\mathrm{c}}\left(t_{j}\right)+\left(\boldsymbol{u}_{\mathrm{c}}\left(t_{j+1}\right)-\boldsymbol{u}_{\mathrm{c}}\left(t_{j}\right)\right)\left(\frac{i \delta t}{\delta t_{\mathrm{th}}}-j\right)
$$

One may note that there exists a time shift $\delta t_{\mathrm{th}}$ between dynamics and thermomechanics. Here again, the convergence analysis carried out in the following with respect to the duration $\delta t_{\mathrm{th}}$, validates a posteriori this strategy.

Note: for the sake of clarity, the time step $\delta t_{\text {th }}$ is denoted $N_{f}$ and is referred to as the thermomechanical update rate in the following. Though this quantity is a duration, it is more conveniently expressed as the number of revolutions the blade makes between two thermomechanical computations.

\section{Convergence}

The proposed numerical strategy relies on a variety of parameters which influence must be carefully assessed in order to ensure that predicted results are relevant. To this end, a validation test case is presented in the first part of this section. This test case features amplified heat fluxes in order to magnify any error or discrepancy that may arise while keeping reasonable computation times. Because a reference solution with full FEM models cannot be obtained (it would require months of computation), an asymptotical convergence of the results is checked for each of the key numerical parameters.

\subsection{Validation test case}

\subsubsection{Contact configuration}

The validation test case features a single low-pressure compressor blade rotating within a deformed casing. The blade angular speed $\Omega$ is constant and belongs to the engine nominal angular speed range. Simulations are performed over 100 blade revolutions. The finite element model of the industrial blade is displayed in Fig. 13a. Clamping conditions are considered at the blade root. Boundary nodes (o) are located along the blade-tip, they are evenly spaced between the leading edge (LE) and the trailing edge (TE). Blade-tip/casing contact is initiated through a radial deformation of the casing, whose circumferential pattern can control a variety of distinct contact scenarios, as evidenced by the chosen profile in Fig. 13b. However, in order to avoid any initial penetration (at $t=0 \mathrm{~s})$ and, the casing is initially perfectly circular. The deformation is progressively applied over the first revolutions of the blade to prevent numerical instabilities detrimental to getting converged results. The initial clearance is $0.1 \mathrm{~mm}$ while the maximum radial distortion of the casing is $1.5 \mathrm{~mm}$. After the application of centrifugal loading, blade-tip/casing clearances increase from the leading edge to the trailing edge. Accordingly, contact is initiated at the blade's leading edge. 


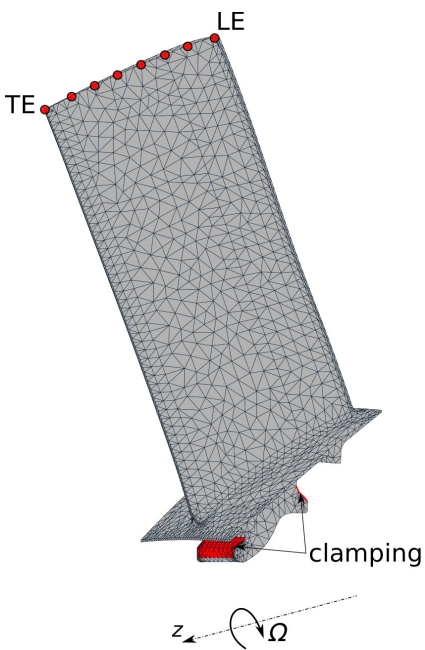

(a) blade finite element model

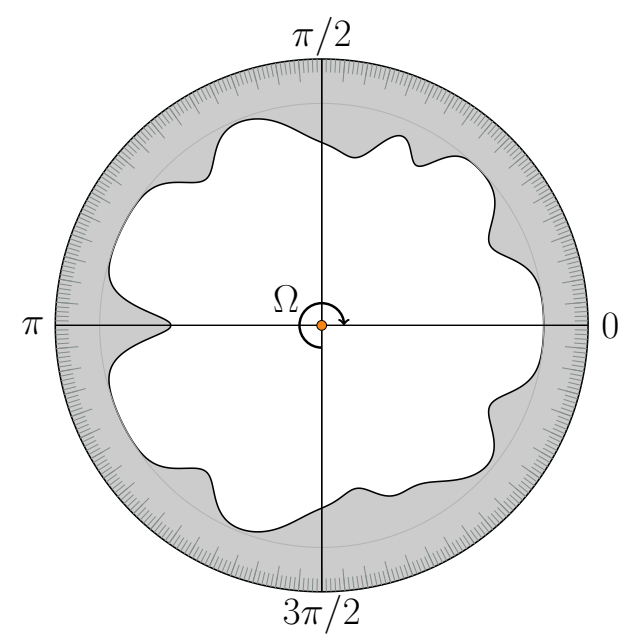

(b) vasing deformation (radial displacements are magnified for the sake of readability)

Figure 13. validation test case

\subsubsection{Model parameters}

The time step of the explicit time integration procedure is set to: $\delta t=10^{-7} \mathrm{~s}$. Five constraint modes are retained in the reduced order model of the blade: $\eta=5$. A single modal damping coefficient is considered for the blade: $\xi=5 \cdot 10^{-4}$. A $3 \mathrm{~mm}$ thick abradable coating is deposited on the casing along its inner surface. Abradable wear is accounted for using a plastic constitutive law previously described in [28]. Mechanical parameters of the abradable coating include its Young's modulus $E=2 \mathrm{GPa}$, plastic modulus $K=0.5 \mathrm{GPa}$ and yield stress $\sigma_{Y}=1.5 \mathrm{~Pa}$. These material properties are purely numerical and may be used to calibrate blade stresses and abradable wear levels. A plastic law featuring a very low yield stress is more appropriate for the calibration on experimental interaction scenarios. A friction coefficient $\mu=0.15$ is considered for contact treatment between the blade and the abradable coating. This choice is motivated by both experimental and numerical investigations. A force ratio $\lambda^{t} / \lambda^{n}=0.15$ has been measured on the ONERA's test bench [27], involving a simplified blade impacting a rotating drum covered with the same abradable material. This value is also close to experimental measurements from abradability tests [35] at room temperature. One may note that this value was also considered in previous publications for the simulation of other interaction scenarios with COROS [22, 24] or with the finite element method [21].

The thermomechanical update of the casing is performed every 10 revolutions, so that $N_{f}=10$. Heat fluxes are magnified by a factor 100. As a result, thermal expansions in the radial direction are amplified and reach about $0.5 \mathrm{~mm}$ over the duration of the simulation. The casing mesh is discretized using $n_{\theta}=1080$ elements along its circumference, and $n_{r}=10$ elements through its thickness. Thermomechanical properties of the casing finite element model are not detailed here for confidentiality reasons.

This standard configuration is used as a reference point for computation times in the following convergence study.

\subsubsection{Quantities of interest}

The goal of the proposed numerical strategy is to accurately capture the casing thermal expansions due to repeated blade-tip/casing contacts. In return, these expansions yield a radial distortion of the casing inner surface which modify the blade/casing contact scenario thus influencing the blade's dynamics. For this reason, it is crucial to assess the convergence of the procedure focusing on quantities related to the casing inner surface, the blade's dynamics and the energy transfer during the simulation:

thermal expansions of the casing: $u_{\mathbf{c}, \mathbf{r}}(t)$. This quantity represents the radial expansions predicted by the thermomechanical simulations along the casing circumference in front of the blade's leading edge. 
blade-tip displacement: $u_{\mathbf{b}, \theta}(t)$. The blade's displacement is considered at the leading edge in the circumferential direction.

energy dissipated by friction: $e(t)$. This is the total energy transferred from the blade to the casing due to the friction. It is related to the heat fluxes summed over the impacted abradable elements: $e(t)=\sum_{\text {elem }} \Sigma \boldsymbol{\varphi}_{\mathrm{ab}} s_{\mathrm{e}} \delta t$.

\subsubsection{Influence of thermal expansions}

It is important to ensure that the proposed validation test case brings forward aspects related to the thermomechanical coupling which is the novelty of the proposed numerical strategy. For each of the three aforementioned quantities of interest, results obtained with ( - ) and without (-) activation of the thermomechanical coupling are depicted in Fig. 14.

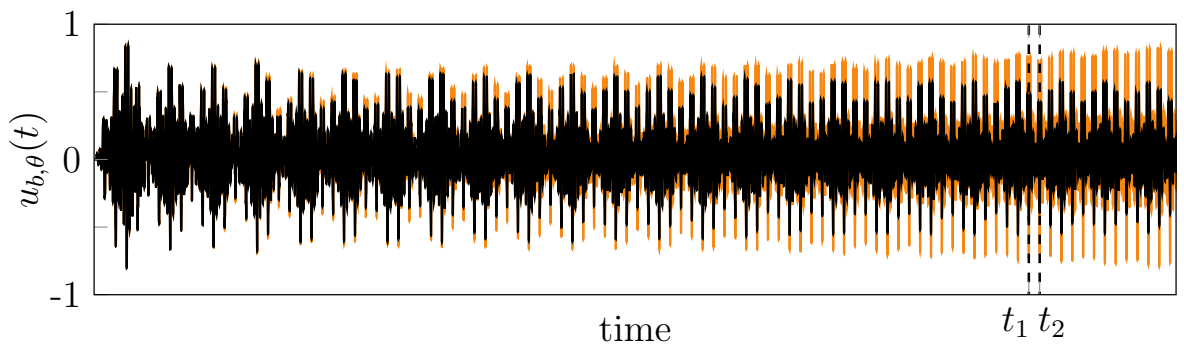

(a) blade circumferential vibration $u_{b, \theta}(t)$

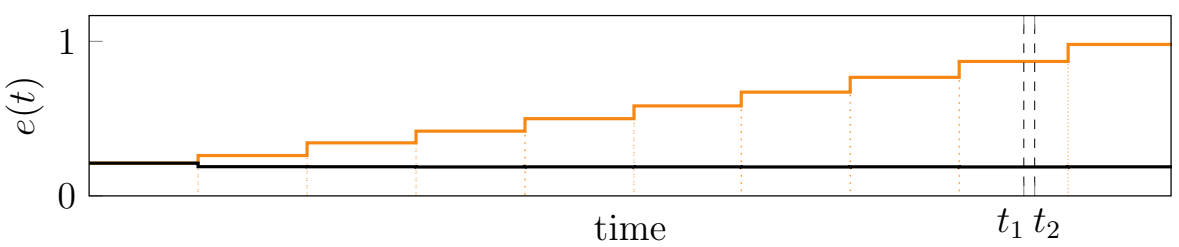

(b) energy dissipated by friction $e(t)$

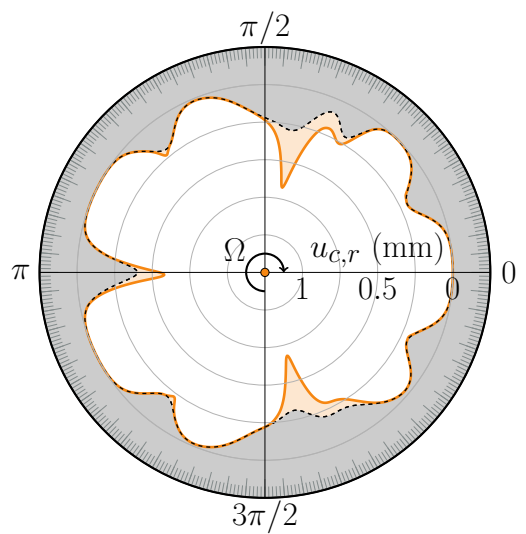

(c) thermal radial expansion $u_{c, r}(t)$

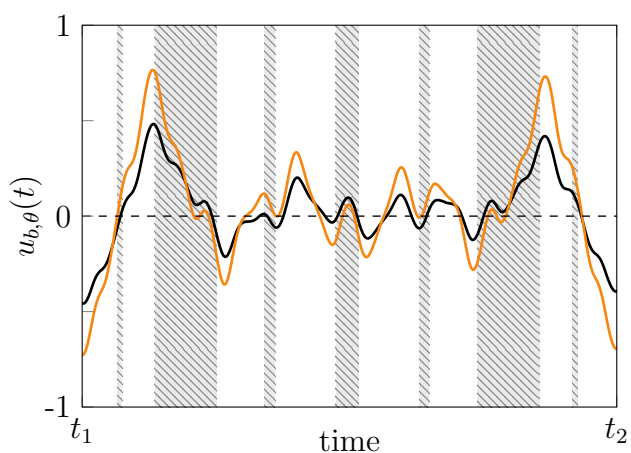

(d) zoom in $u_{b, \theta}(t)$

Figure 14. results of the benchmark case with (_) and without ( - ) thermomechanical coupling

Without thermomechanical coupling, the abradable is worn off during the very first blade/casing contacts. During the subsequent revolutions, see $u_{b, \theta}(t)$ in Fig. 14a, a steady state is reached: amplitudes of vibration remain constant. Similarly, the energy transferred to the casing $e(t)$ quickly stabilizes around a constant value.

However, when thermomechanical effects are accounted for, thermal expansions on the casing inner surface yield a very different contact scenario: the friction energy $e(t)$ transferred to the casing increases over time. Also, the 
amplitude of $u_{b, \theta}(t)$ increases over time, see Fig 14a. A zoom over a narrow time frame corresponding to one blade revolution is provided in Fig. 14d. Hatched areas are used to represent instants over which the blade is in contact with the casing. Finally, accounting for thermomechanical effects yield some radial expansion of the casing contact surface that are depicted in Fig. 14c.

\subsection{Time convergence}

Time convergence refers to the numerical behavior of the proposed strategy as time integration parameters, such as the different time steps, vary.

\subsubsection{Thermomechanical update rate: $N_{f}$}

As mentioned above, $N_{f}$ represents the time span between two thermomechanical simulations. It is here expressed as a number of blade revolutions. Due to the high computational cost associated with the FEM simulations, the choice of $N_{f}$ is strategic as it significantly impacts the overall efficiency of the numerical procedure. On average, every call to the thermomechanical Ansys procedure costs about 13 minutes in computation times. Simulations are performed with different values of $N_{f}$ from 1 to 20. The aforementioned quantities of interest are plotted in Fig. 15. $N_{f}$ has low to no influence on the blade vibration as it is noticeable that plots of $u_{b, \theta}$ obtained for each value of $N_{f}$ are almost perfectly superimposed: differences only appear for the highest value $N_{f}=20$, see Fig. $15 \mathrm{c}$. The same observation can be made with respect to the thermal expansions of the casing, see Fig. 15b. To the contrary, $N_{f}$ has a strong influence on the energy $e(t)$ transferred from the blade to the casing during the interaction. Indeed, as depicted in Fig. 15a, it is evidenced that a high value of $N_{f}$ leads to significant cumulated errors over time. Logically, smaller values of $N_{f}$ provide higher resolution of $e(t)$ to a point that its variation during transient phases may be seen for $N_{f}=1$ at the beginning of the simulation.

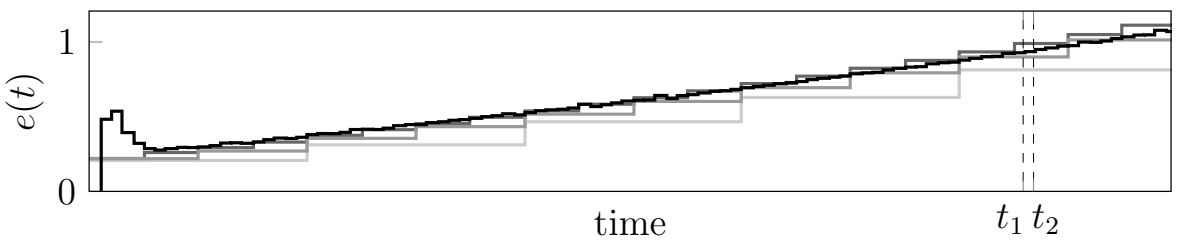

(a) transferred energy $e(t)$

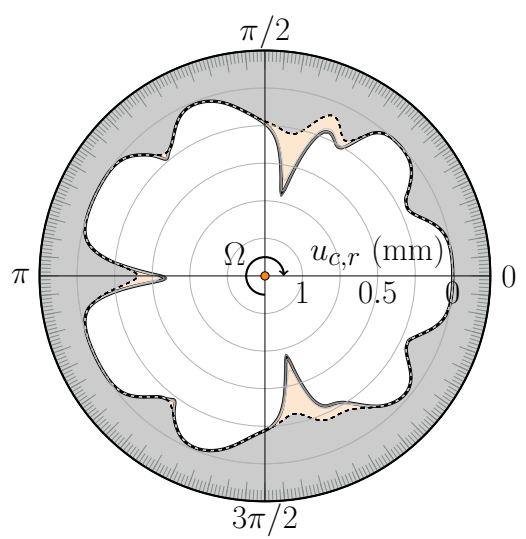

(b) thermal radial expansion $u_{c, r}(t)$

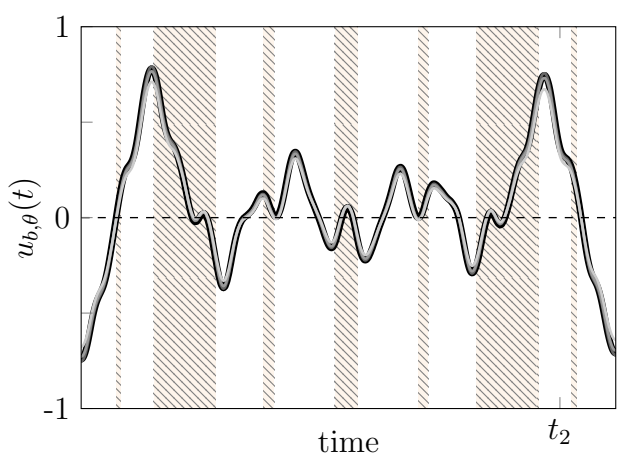

(c) blade circumferential vibration $u_{b, \theta}(t)$

Figure 15. quantities of interest obtained for $N_{f}=20(-), 10(-), 5(-), 1(\square)$

Putting aside transient phenomena that can only be captured for $N_{f} \leq 1$, there is a good superimposition of the obtained results as $N_{f}$ decreases. Computation times - all simulations are carried out on a standard i7-processor (i7-7700K - quad core $4.20 \mathrm{GHz}$ ) personal computer - obtained for each $N_{f}$ are listed in Tab. 1. These computation times are relative to the aforementioned standard configuration featuring $N_{f}=10$. 


\begin{tabular}{|l|c|c|c|c|}
\hline$N_{f}$ & 1 & $\mathbf{5}$ & 10 & 20 \\
\hline CPU time & $+480 \mathrm{~min}$ & $+105 \mathrm{~min}$ & $\mathbf{1 5 0} \mathbf{~ m i n}$ & $-70 \mathrm{~min}$ \\
\hline
\end{tabular}

Table 1. computation times for different values of $N_{f}$

In view of result accuracies and gains of time, the value $N_{f}=10$ is considered in the following.

\subsubsection{Dynamic time step: $\delta t$}

The expression dynamic time step refers to the time integration step of the contact management with COROS. Previous investigations carried out with the same contact management procedure underlined that typical values of $\delta t$ are in the vicinity of $10^{-7} \mathrm{~s}$. For this reason, investigated values of $\delta t$ here range from $10^{-8} \mathrm{~s}$ to $2 \cdot 10^{-7} \mathrm{~s}$. Time steps larger than $2 \cdot 10^{-7} \mathrm{~s}$ yield numerical divergence of the simulation.

The three quantities of interest are depicted in Fig. 16 for the different values of $\delta t$. It is visible that all plots are perfectly superimposed. The much longer time scale of thermomechanical effects logically makes the thermomechanical simulation insensitive to $\delta t$.

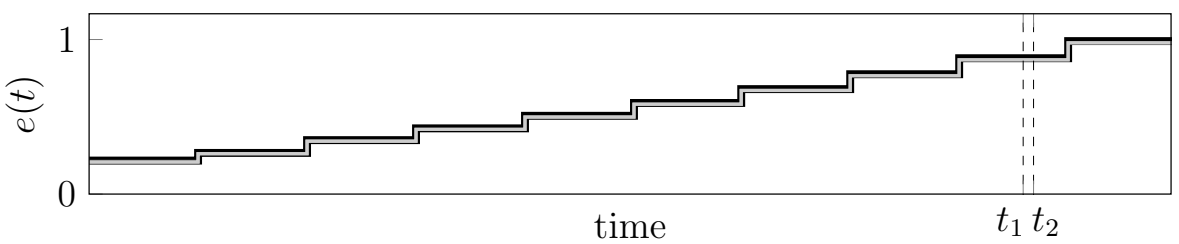

(a) transferred energy $e(t)$
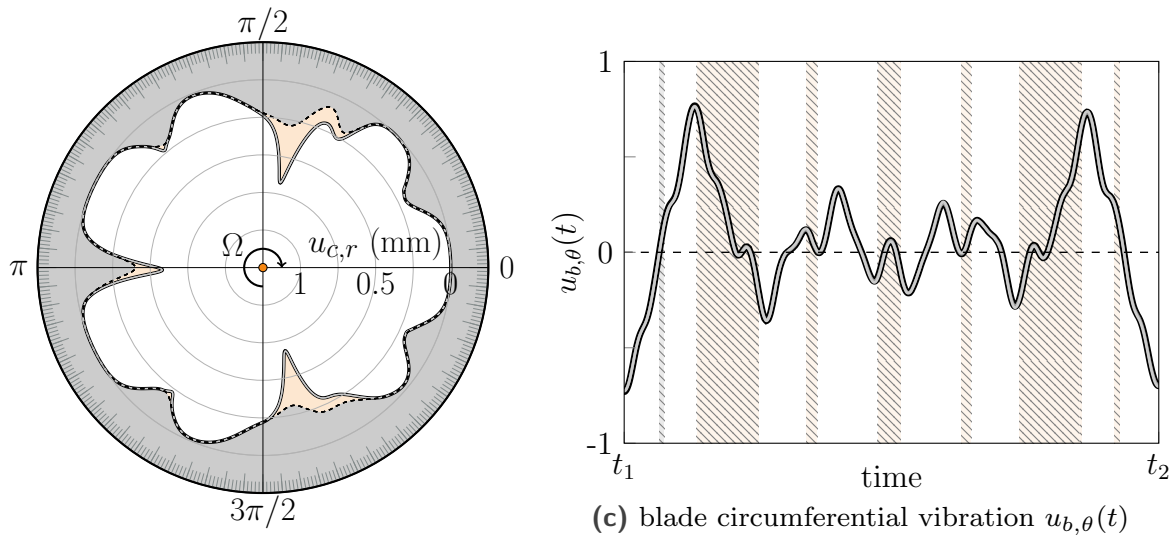

(c) blade circumferential vibration $u_{b, \theta}(t)$

(b) thermal radial expansion $u_{c, r}(t)$

Figure 16. quantities of interest obtained for $\delta t=10^{-8} \mathrm{~s}(-), 5 \cdot 10^{-8} \mathrm{~s}(\square), 10^{-7} \mathrm{~s}(\longrightarrow), 2 \cdot 10^{-7} \mathrm{~s}(\longrightarrow)$

Computation times obtained as $\delta t$ varies are listed in Tab. 2. The relatively small impact of $\delta t$ on the overall computation times (only $+15 \%$ when $\delta t$ is divided by 2 from $10^{-7} \mathrm{~s}$ to $5 \cdot 10^{-8} \mathrm{~s}$ ) underlines that COROS computational cost is low in comparison to the thermomechanical procedure.

\begin{tabular}{|l|c|c|c|c|}
\hline$\delta t$ & $10^{-8} \mathrm{~s}$ & $5 \cdot 10^{-8} \mathrm{~s}$ & $10^{-7} \mathrm{~s}$ & $2 \cdot 10^{-7} \mathrm{~s}$ \\
\hline CPU time & $+40 \mathrm{~min}$ & $+23 \mathrm{~min}$ & $\mathbf{1 5 0} \mathbf{~} \mathbf{m i n}$ & $-8 \mathrm{~min}$ \\
\hline
\end{tabular}

Table 2. computation times for different values of $\delta t$ 


\subsubsection{Thermal time step:}

The convergence with respect to the thermal time step has also been carefully checked. This quantity refers to the numerical time step of the transient thermal simulations carried out using Ansys. Results are only briefly summed up here for the sake of brevity. Differents values were investigated, with low to no impact on the results: a perfect superimposition of the results was found for each quantity of interest.

\subsection{Space convergence}

The notion of space convergence refers to all the numerical parameters that are related to space discretization such as mesh sizes of the employed finite element meshes.

\subsubsection{Number of elements in the circumference: $n_{\theta}$}

Within COROS, parameter $n_{\theta}$ refers to the number of abradable elements along the casing contact surface in the tangential direction. It also corresponds to the number of finite element of the casing along the tangential direction within the thermomechanical procedure.

Results obtained for $n_{\theta}$ ranging from 360 to 2,160 are shown in Fig. 17. The lowest value of $n_{\theta}$ yields significant errors in terms of thermal expansions, blade-tip displacements and energy transferred to the casing. However, increasing $n_{\theta}$ leads to a prompt convergence of both thermal expansions and blade-tip displacements. The asympotic convergence of the results as $n_{\theta}$ increases is also evidenced in terms of the energy transferred to the casing, see Fig. 17a.

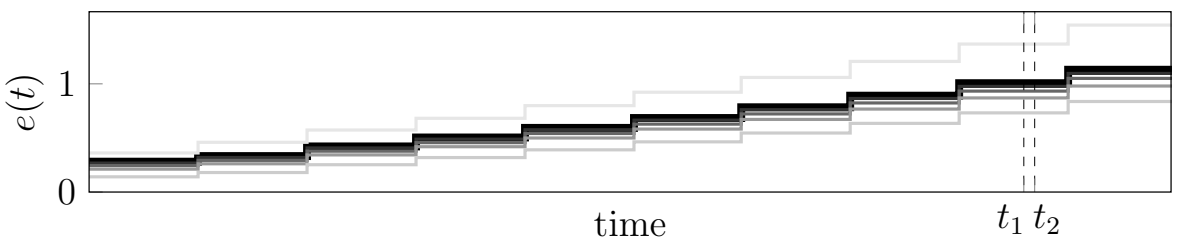

(a) transferred energy $e(t)$

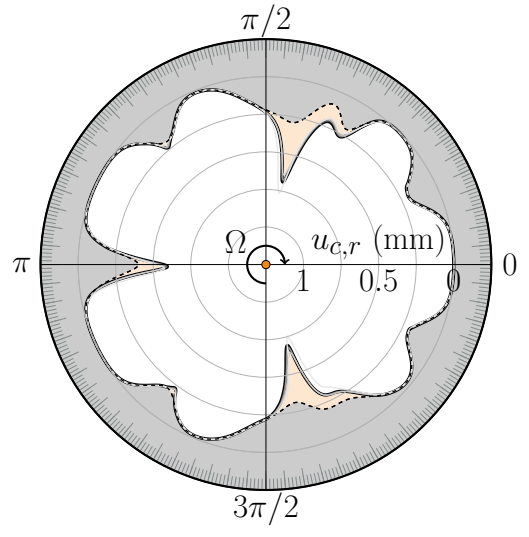

(b) thermal radial expansion $u_{c, r}(t)$

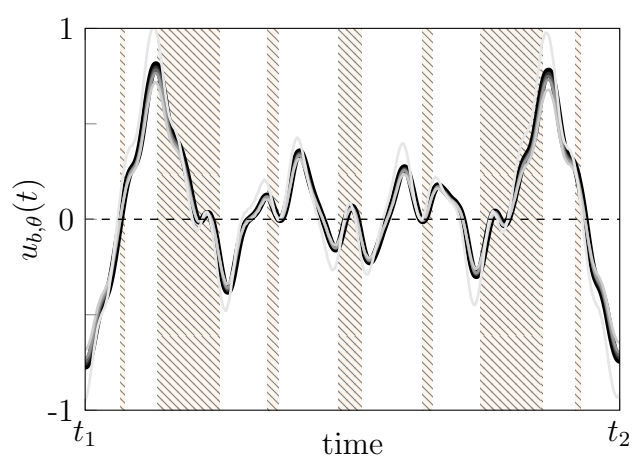

(c) blade circumferential vibration $u_{b, \theta}(t)$

Figure 17. quantities of interest obtained for $n_{\theta}=360(-), 720(-), 1080(-), 1440(\square), 1800(\square), 2160(\longrightarrow)$

Computation times for the considered values of $n_{\theta}$ are listed in Tab. 3. It is noticeable that there is a significant cost to increasing the value of $n_{\theta}$. For this reason, a compromise must be found between accuracy and computational cost.

The value $n_{\theta}=1,080$ offers the best compromise as it allows to predict very accurately both blade-tip displacements and thermal expansions. This value is considered for all the numerical simulations presented in the following. 


\begin{tabular}{|l|c|c|c|c|c|c|}
\hline$n_{\theta}$ & 2160 & 1800 & 1444 & $\mathbf{1 0 8 0}$ & 720 & 360 \\
\hline $\mathrm{CPU}:$ & $+120 \mathrm{~min}$ & $+90 \mathrm{~min}$ & $+30 \mathrm{~min}$ & $\mathbf{1 5 0} \mathrm{min}$ & $-60 \mathrm{~min}$ & $-135 \mathrm{~min}$ \\
\hline
\end{tabular}

Table 3. computation times for different values of $n_{\theta}$

\subsubsection{Number of radial elements: $n_{r}$}

Parameter $n_{r}$ refers to the number of finite elements of the casing through its thickness, along the radial direction. Thus, this parameter is solely related to the thermomechanical procedure. Results are only briefly mentioned here for the sake of brevity. It is found that any value $n_{r} \geq 10$ does not significantly influence any of the three quantities of interest: results are perfectly superimposed. In fact, $n_{r}$ may only influence the computation of local quantities such as temperatures in the vicinity of impacted areas, for which there is no experimental data. Accordingly, in the context of the presented investigation, $n_{r}=10$ is deemed satisfactory and is employed in the following.

\subsubsection{Reduction parameter: $\eta$}

The reduction parameter $\eta$ controls the number of constraint modes that are retained within the Craig-Bampton [36] based component mode synthesis technique used for building the blade's reduced order model. The convergence of the results obtained with the proposed numerical strategy with respect to $\eta$ is extremely fast. It is found that results related to the three quantities of interest are perfectly superimposed for any value of $\eta \geq 2$. This is consistent with the fact that the employed Craig-Bampton based reduction technique is known to provide a very accurate representation of the boundary nodes displacements that are intimately related to the three quantities of interest. In order to ensure an optimal representation of the blade dynamics, $\eta=5$ is considered in the following.

\subsection{Summary}

Based on the convergence analysis carried out for each numerical parameter, the simulation of the experimental configuration is done using:

- thermomechanical update rate: $N_{f}=10$ revolutions,

- dynamic time step: $\delta t=10^{-7} \mathrm{~s}$,

- $n_{\theta}=1,080$ and $n_{r}=10$ for the casing finite element model,

- reduction parameter: $\eta=5$.

The definition of other numerical parameters comes from empirical data:

- abradable mechanical properties: $E=2 \mathrm{GPa}, K=0.5 \mathrm{GPa}$ and $\sigma_{Y}=1.5 \mathrm{~Pa}$,

- friction coefficient: $\mu=0.15$

- blade modal damping coefficient: $\xi=5 \cdot 10^{-4}$

\section{Numerical simulation of the experimental configuration}

The proposed multi-physics numerical strategy is here applied to the experimental contact scenario presented in section 2.

Note: for confidentiality reasons, some quantities are normalized in the following.

\subsection{Description of the contact scenario}

Experimental data and observations are used to define a numerical contact scenario as close as possible to the experimental rubbing event. Particular attention is paid to recreate the fairly low incursion depth of the blade within the abradable coating, it is here set to $50 \mu \mathrm{m}$. In agreement with experimental measurements, the casing is represented by a perfectly rigid profile. The variation of this profile along the tangential direction however remains uncertain as only very limited experimental data are available. A perfectly ovalized profile is here assumed, with a maximum radial distortion of $450 \mu \mathrm{m}$ that matches experimental measurements [21] and yields the aforementioned incursion depth. This ovalization is progressively applied over the first revolutions of the blade in order to initiate contact. 
Contact simulations are carried out considering a constant angular speed $\Omega$. Several values of $\Omega$ are investigated in order to identify the numerical critical speed. This critical speed is $\Omega^{*}=1.15 \Omega_{h 7}$. The discrepancy between experimental and numerical critical angular speeds can be explained by several factors including: (1) the use of perfectly clamped boundary conditions on the blade finite element model, (2) an unavoidable discrepancy between the actual blade first two eigenfrequencies and the values computed for a perfect CAD model, (3) a linearized procedure to account for centrifugal stiffening and, (4) a simplified representation of the casing that is initially perfectly ovalized in the numerical model.

\subsection{Simulation without thermomechanics}

A simulation is first run without thermomecanical effects using COROS alone. The simulated interaction lasts 47 seconds, which corresponds to more than a thousand blade revolutions. The predicted circumferential displacement of the blade's leading edge is pictured in Fig. 18a, along with a zoom over one blade revolution in Fig. 18b. The wear profile at the leading edge at the end of the simulation is plotted in Fig. 18c along with a zoom over the angular position $\pi / 2$ in Fig. 18d.

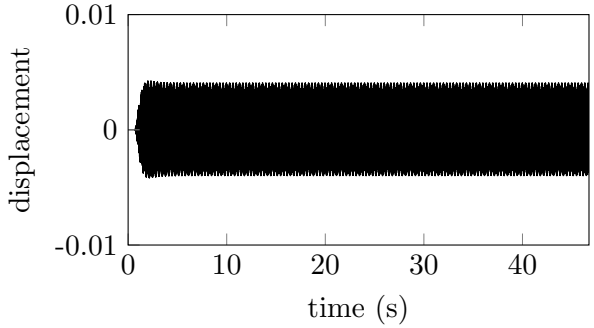

(a) leading edge circumferential vibration

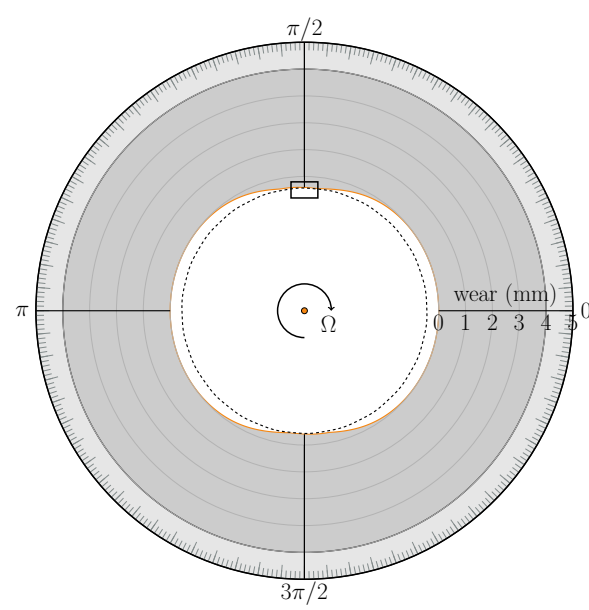

(c) wear profile at the leading edge at $t=47 \mathrm{~s}$ and infront radial trajectory of the blade in the previous revolution

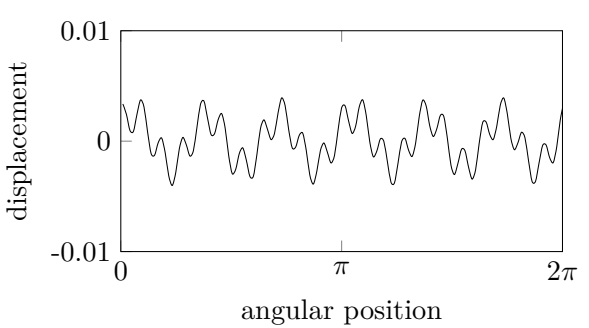

(b) zoom over a revolution at $t=47 \mathrm{~s}$

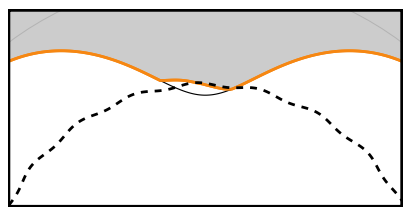

(d) zoom over the angular position $\pi / 2$

Figure 18. numerical procedure's results without thermomecanics

Following contact initiation, amplitudes of vibration increase as repeated contacts occur and the ovalization of the casing is established. Once the casing is ovalized, for about $t>3 \mathrm{~s}$, amplitudes of vibration remain constant with time: after a certain amount of abradable coating is worn off, a steady state is reached where the coating undergoes purely elastic deformations. The amount of abradable coating worn off at the end of the simulation is of the same order of magnitude as the incursion depth: $50 \mu \mathrm{m}$. Worn off areas are located at $\theta=\pi / 2$ and $\theta=3 \pi / 2$ 
where the ovalization of the casing yields negative clearances. The blade-tip trajectory during the last revolution (- - - ) is superimposed to the predicted wear profile at the end of the last revolution (- ${ }^{-}$in Fig. 18c.

These results underline that the very low incursion depth of the blade within the abradable coating does not yield any divergent behaviour in terms of amplitudes of vibration. Finally, though results presented in this section are obtained for $\Omega^{*}$, it has been checked that presented conclusions remain valid in the vicinity of $\Omega^{*}$ where no significant amplitudes of vibration are predicted.

\subsection{Multi-physics numerical simulation}

The thermomechanical procedure is now coupled to COROS. Obtained results are presented both in time and frequency domain in order to provide an in-depth analysis of the simulated interaction. In particular, access to the abradable coating wear levels at key instants of the interaction provides new insight with respect to the type of phenomena that may trigger the burst of amplitudes observed experimentally.

\subsubsection{Blade vibration analysis}

The blade's leading edge circumferential displacement is plotted in Fig. 19. It is noticeable that the simulated interaction is divergent. For that reason, particular attention must be paid to the blade amplitudes of vibration in order to make sure that predicted results fall within the validity domain of the model. The frontier of the small perturbation framework (-- ) is plotted in Fig. 19. It corresponds to a blade-tip circumferential displacement equal to $10 \%$ of the blade length. Above this critical threshold, the maximum stress in the blade exceeds the blade's yield stress $\sigma_{y}$. It is assumed that beyond this value, which is reached for $t \geq 47 \mathrm{~s}$, obtained results are not valid. Consequently, the analysis of the results is limited to the time window $t \in[0,47] \mathrm{s}$.

While contact is initiated during the very first revolutions of the blade, amplitudes of vibration remain very low for about 35 seconds. Nonetheless, a regular increase of the amplitudes of vibration is noticeable over this period of time. For $t \in[35,45] \mathrm{s}$, amplitudes of vibration become more significant before a sudden and brutal burst after $t=45 \mathrm{~s}$. Accordingly, based on the time response depicted in Fig. 19, three phases may be distinguished during the

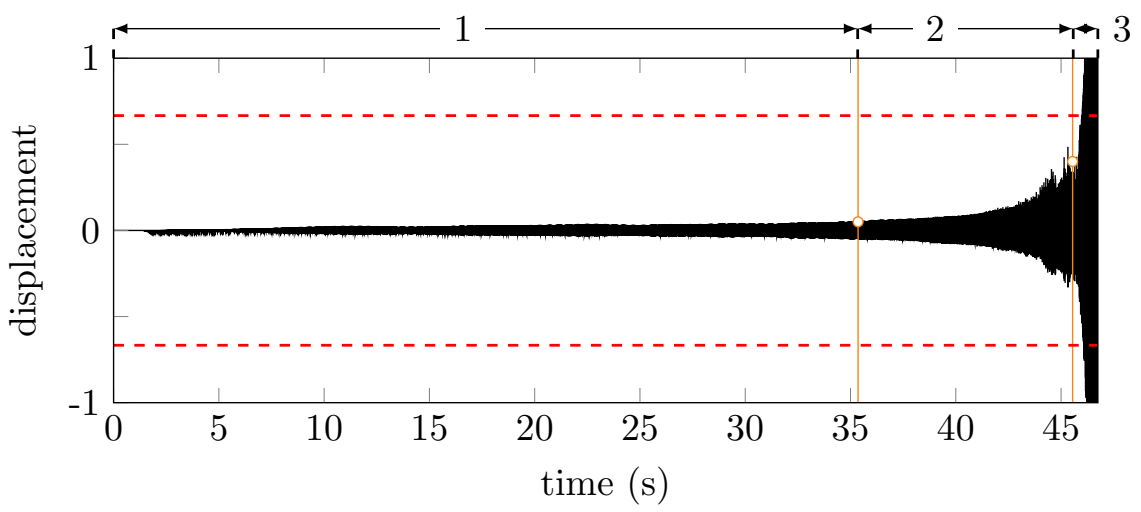

Figure 19. vibration of the blade's leading edge in the circumferential direction

interaction, namely: phase 1 for $t \in[0,35] \mathrm{s}$, phase 2 for $t \in[35,45] \mathrm{s}$ and phase 3 for $t \geq 45 \mathrm{~s}$.

A spectrogram of the blade's leading edge circumferential displacement is given in Fig. 20a. The three aforementioned phases are identified using orange lines. At the angular speed $\Omega^{*}$, each engine order corresponds to a certain frequency of excitation. For the sake of simplicity, these engine orders are directly marked along the ordinate axis of Figs. 20. In the frequency domain, a distinct harmonic content is found for each phase. During phase 1, two dominant frequencies are found in the vicinity of the engine orders $e_{o 6}$ and $e_{o 22}$.

During the second phase of the interaction, amplitudes of vibration evidenced along $e_{o 22}$ disappear as amplitudes along $e_{06}$ increase. After a while, maximum amplitudes of vibration are found between $e_{06}$ and $e_{07}$, right above the eigenfrequency of the first bending mode, see Fig. 20b. 


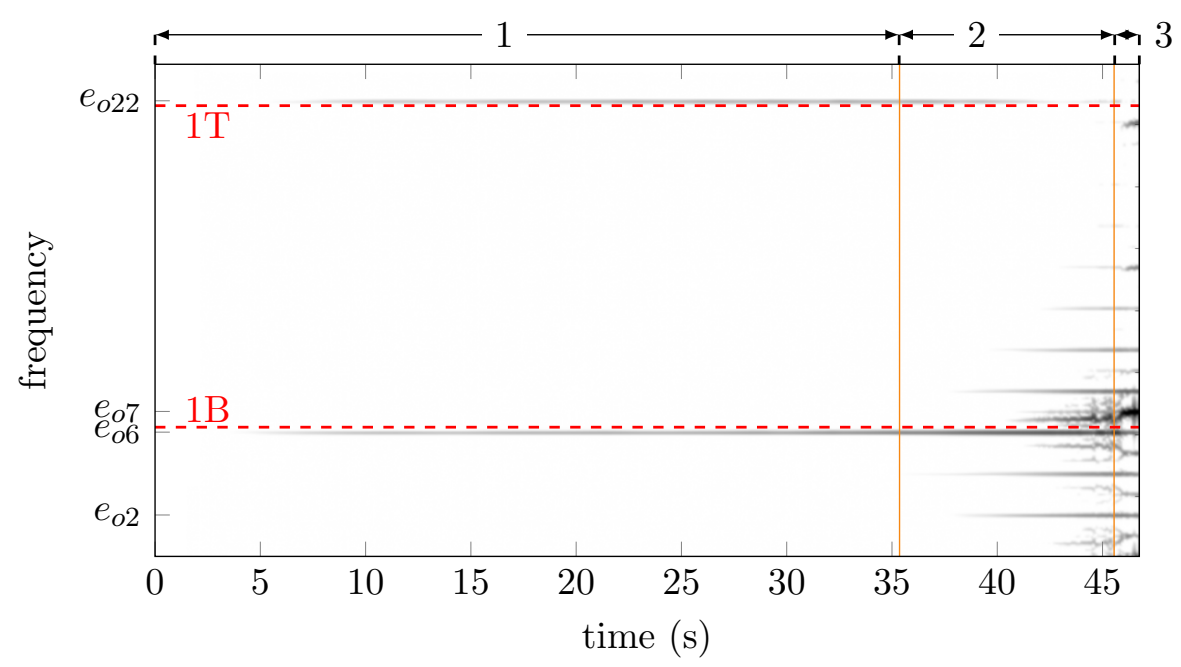

(a) full spectrogram

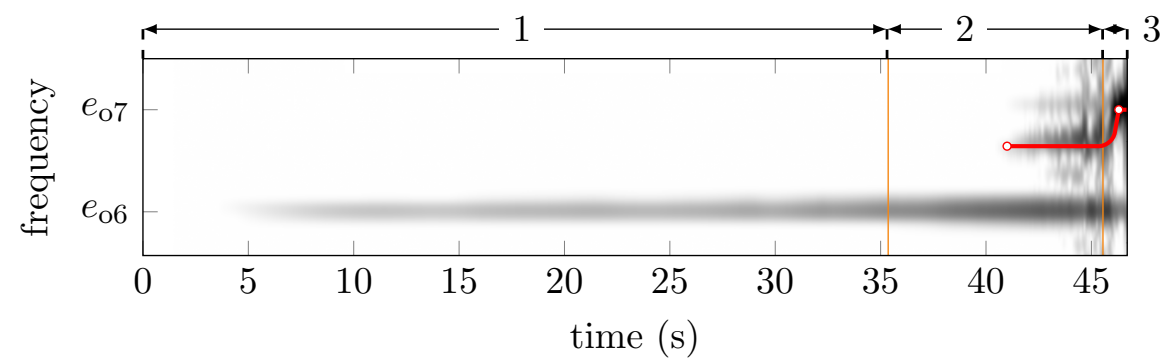

(b) zoom on $e_{06}$ and $e_{07}$

Figure 20. spectrogram of blade's leading edge circumferential displacement

Interestingly, the transition from phase 2 to phase 3 is marked by a sudden shift of the peak of amplitudes found between $e_{06}$ and $e_{07}$, see the added red line (-) in Fig. 20b. The frequency shift is such that the peak merges with $e_{07}$ at the precise moment where amplitudes of vibration diverge. There is no doubt that the predicted interaction essentially involves the first bending mode of the blade since amplitudes found for engine orders above $e_{o 8}$ apart multiples of seven, do not increase significantly during phase 3 .

Finally, the employed numerical methodology allows to obtain stress fields within the blade at any time step. Three stress fields are plotted in Fig. 21, corresponding respectively to the end of phases 1, 2 and 3. The colour code used for each of this field is identical to facilitate direct comparison. These stress fields are computed at a time where amplitudes of vibration are maximum (marked with white dots in Fig. 19). At the end of phases 1 and 2, maximum stresses are found in the middle of the blade root as well as along the leading edge, close to the root thus suggesting a similar vibration pattern. At the end of phase 3 however, maximum stresses are computed along the leading edge, at about a quarter of the blade height (see area A in Fig. 21c). Area A precisely corresponds to a damaged area on the experimental blade [21]. One may note that the maximum stress computed in the blade at the end of phase 3 corresponds to the yield strength $\sigma_{y}$ of the blade material. This peak value indicates that: (1) before $t=46 \mathrm{~s}$, stresses within the blade are such that the blade remains within its elastic domain thus confirming the validity of the carried out simulation and, (2) area A is a privileged location for crack initiation, in agreement with experimental observations. 


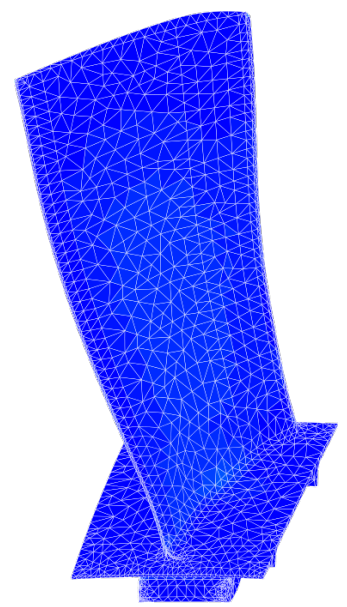

(a) $t=35 \mathrm{~s}$

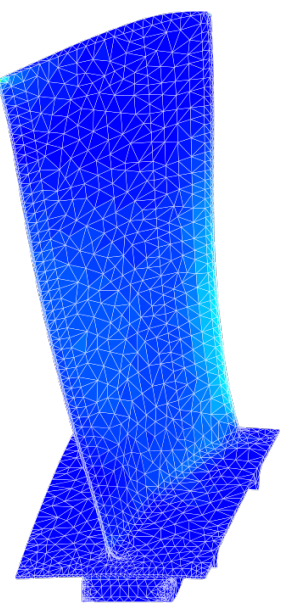

(b) $t=45 \mathrm{~s}$

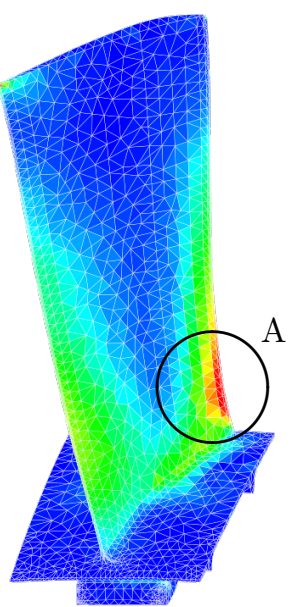

(c) $t=47 \mathrm{~s}$

0

$\sigma_{y} / 2$

(d) colour code

Figure 21. blade stress fields

\subsubsection{Predicted wear patterns}

The employed numerical strategy provides access to abradable wear levels and thermal expansions at any time step. In this section, the combination of thermal expansions and wear is plotted in polar coordinate in orange ( - ). The trajectory of the corresponding blade contact node over one revolution is superimposed in black (- - - ). When needed, the trajectory of the same node over previous revolutions is indicated in grey. These quantities are plotted at the end of each phase in Figs. 22, 23 and 24.

At the end of phase 1 , contact areas are located at $\theta=\pi / 2$ and $\theta=3 \pi / 2$. Repeated contacts at the leading edge lead to temperatures increase and localized thermal expansions that allow to initiate contact at the trailing edge. The establishment of contact at the trailing edge marks the end of phase 1 .

At the end of phase 2, non-synchronous vibrations are evidenced at the trailing edge. Indeed, it may be seen in Fig. 23b that trajectories of the blade contact node over the last two revolutions are circumferentially shifted. Wear levels at the trailing edge become significant, up to $1 \mathrm{~mm}$. Meanwhile, the blade amplitudes of vibration at the leading edge remain low with wear areas still mostly located around $\theta=\pi / 2$ and $\theta=3 \pi / 2$, see Fig. 23a.

Predicted wear patterns at the end of phase 3, depicted in Fig. 24, underline that the blade dynamics is now synchronous with seven clearly visible wear lobes at the trailing edge. It is remarkable that no significant wear is predicted at the leading edge: the blade growing amplitudes of vibration essentially involve its trailing edge.

A spatial Fourier transform of predicted wear profiles at the end of each phase is plotted in Fig. 25. At the end of phase 1, wear patterns and thermal expansions mostly exhibit a frequency content related to harmonics multiple of 2 due to the casing ovalization. At the end of phase 2 however, harmonic 6 is clearly dominant in agreement with the fact that the blade dynamics is mostly driven by $e_{o 6}$. Finally, at the end of phase 3 , the seven deep wear lobes naturally lead to a clear dominant harmonic 7 .

\subsubsection{Energy and temperature levels}

The energy $e(t)$ transferred to the casing during the interaction is depicted in Fig. 26. The envelope of this plot is consistent with the one of the blade displacement depicted in Fig. 19: a very small increase is noticeable over phase 1 and the amplitude explodes during phase 3 .

Temperatures numerically predicted at the end of each phase on the outer surface of the casing, in front of the 


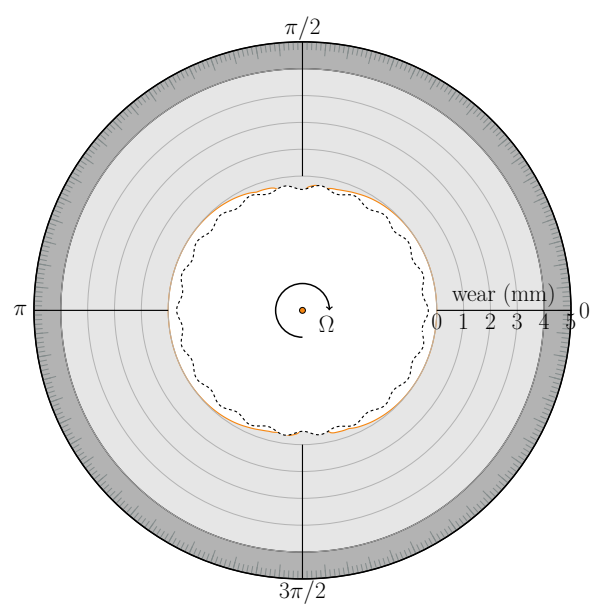

(a) leading edge

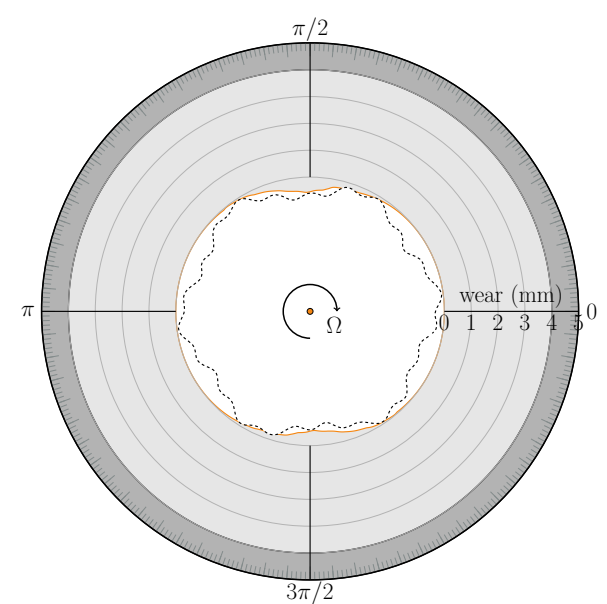

(b) trailing edge

Figure 22. abradable coating inner surface $(-)$ and blade contact node trajectory $(-\mathbf{- ~ - ~})$ at the end of phase $1(t=35 \mathrm{~s})$

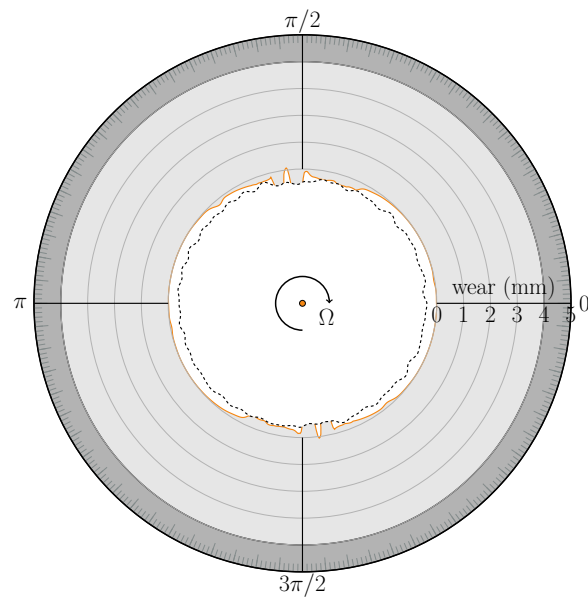

(a) leading edge

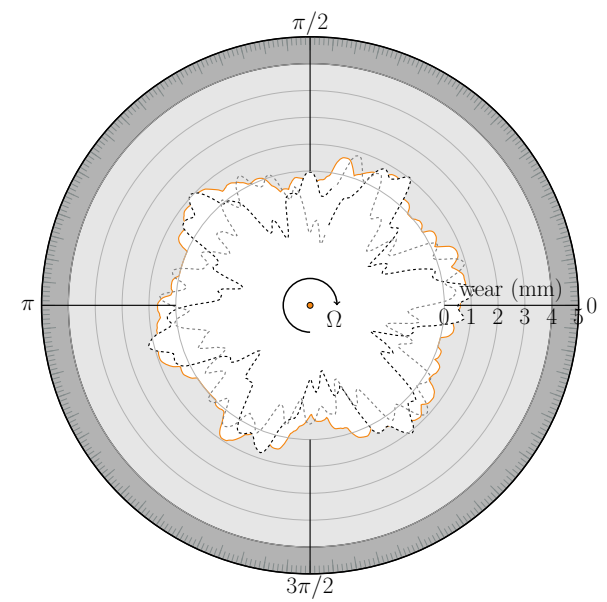

(b) trailing edge

Figure 23. abradable coating inner surface ( -$)$ ) and blade contact node trajectory $(\mathbf{- ~ - ~ - ~})$ at the end of phase $2(t=45 \mathrm{~s})$

middle of chord of the blade, are depicted in Fig. 27. It is found that temperatures increase are very localized, particularly around $\theta=\pi / 2$ and $\theta=3 \pi / 2$ where, due to the casing ovalization, contact is initiated. Experimental measurements are indicated in Fig. 27 by means of orange dots (०). Based on the presented results, it seems that the location of the temperature probes did not allow to capture highest temperature increases since none of them were located in the vicinity of $\pi / 2$ and $3 \pi / 2$. The limited number of experimental measurements makes it arduous to assess the validity of numerical predictions. Nonetheless, it seems that the numerically predicted orders of magnitude of temperatures are in agreement with experimental measurements.

\subsubsection{Assessment of the numerical/experimental confrontation}

Overall, the numerical model provides results that are in line with experimental observations. Predicted wear lobes on the casing are consistent with post-mortem experimental measurements: there is no significant wear predicted at the leading edge and seven deep wear lobes are predicted at the trailing edge, see Figs. 24b and 5c. Also, in terms of 


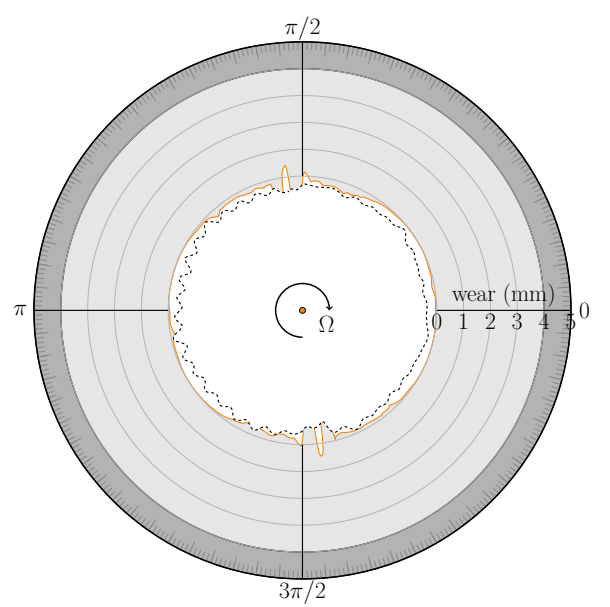

(a) leading edge

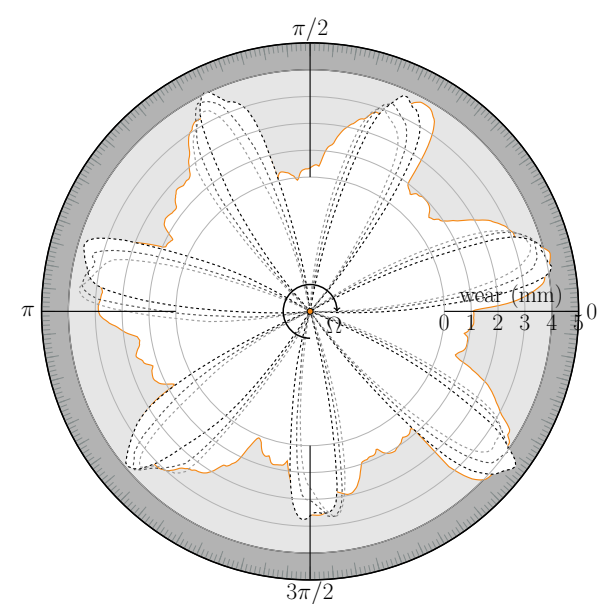

(b) trailing edge

Figure 24. abradable coating inner surface $(-)$ and blade contact node trajectory $(-\mathbf{- ~ - ~})$ at the end of phase $3(t=47 \mathrm{~s})$

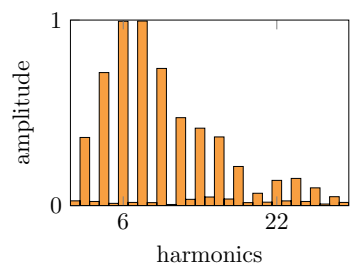

(a) end of phase 1

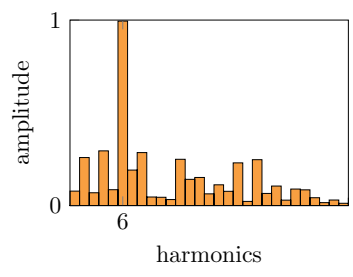

(b) end of phase 2

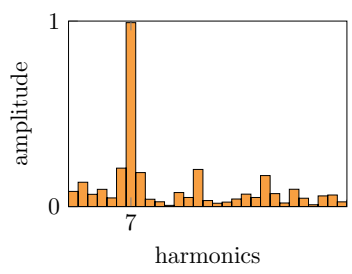

(c) end of phase 3

Figure 25. normalized spatial Fourier transforms of the abradable coating inner surface at the trailing edge

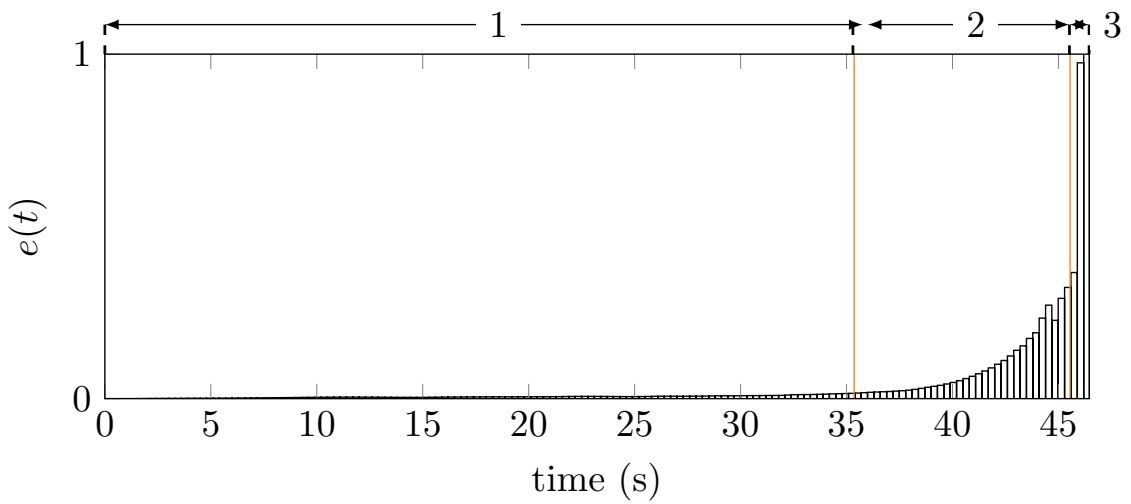

Figure 26. energy transferred to the casing due to friction: $e(t)$

blade dynamics, maximum computed stresses within the blade are consistent with the blade material yield strength and are predicted where cracks were experimentally observed.

From a more qualitative point of view, it is noticeable that the numerically predicted interaction features three distinct phases in agreement with experimental observations. The features of each phase are aligned with experimental findings: a long first phase over which amplitudes of vibration increase slowly, followed by a second shorter phase with higher amplitudes of vibration which started to rise before a third very short phase during which 


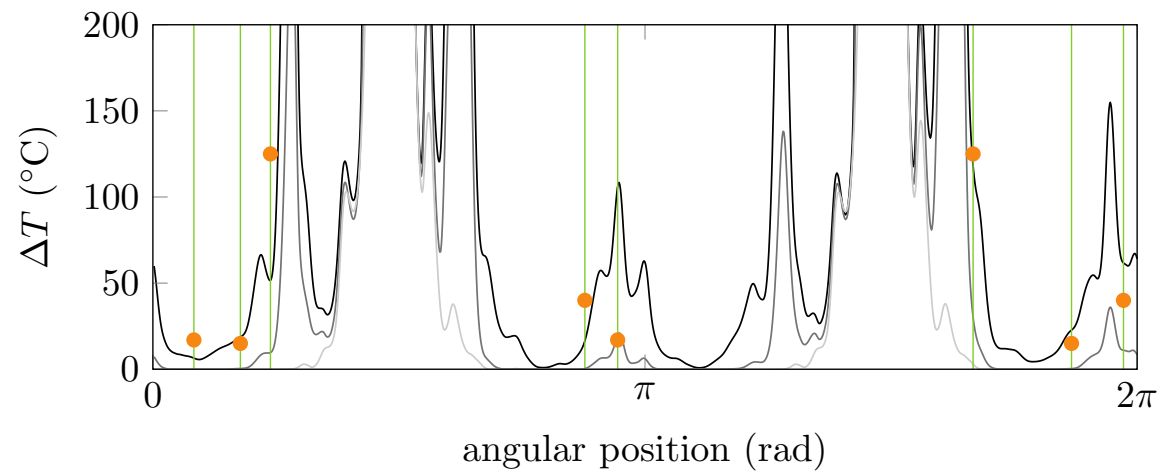

Figure 27. temperature along the outer surface of the casing, facing the middle of chord, at the end of phase 1 ( $\longrightarrow$ ), phase 2 $(-)$ and phase $3(-)$. Angular position of experimental sensors $(-)$ and experimental values $(\circ)$

amplitudes of vibration explode.

The most noticeable difference between experimental observations and numerical predictions is related to the blade vibration behaviour. Indeed, the spectrogram pictured in Fig. 4a underlines that the blade vibration frequency content is driven by $e_{07}$ and $e_{o 25}$ while numerically, it is found that the blade vibration frequency content is mostly located along $e_{o 6}$ and $e_{o 22}$, see Fig. 20a. The shift from engine order $e_{o 25}$ to $e_{o 22}$ may indicate a discrepancy between the second eigenfrequencies of the experimental blade and the associated numerical model. This is most likely due to the use of simplified boundary conditions in the numerical model. With respect to the shift from $e_{o 7}$ to $e_{06}$ during phase 1 , same as for mode 1T, the use of simplified boundary conditions on the blade finite element model may yield a small deviation between the first eigenfrequencies of the experimental blade its numerical model. However, since both experimental observations and numerical predictions yield a divergence along $e_{07}$, it is unlikely that this discrepancy be significant. The shift from $e_{07}$ to $e_{o 6}$ may thus have two explanations: (1) the casing ovalization is numerically perfectly symmetric which promotes a blade dynamics driven by even engine orders. Unfortunately, the lack of experimental data makes it impossible to better describe the casing distortion. Assuming a non symmetric casing distortion would certainly prevent a dominant $e_{o 6}$. (2) It is also possible that the numerical strategy inaccurately predicts the contact stiffening phenomenon, which is also extremely arduous to predict experimentally. Assuming the numerical contact stiffening is underestimated, it is conceivable that an accurate description of the experimental blade dynamics is reached once the level of energy within the mechanical system is sufficiently high, meaning that the experimental conditions of the interaction may only be predicted once contact forces - inherently related to the abradable coating mechanical properties - are high enough thus explaining the shift from $e_{o 6}$ to $e_{07}$ at the end of phase 2 in the numerical model.

What is key however, is that the predicted blade dynamics does involve the same free vibration modes - modes $1 \mathrm{~B}$ and $1 \mathrm{~T}$ - both numerically and experimentally.

Finally, similarities between the experimental set-up and numerical predictions related to temperature levels are assumed less relevant in the sense that experimental measurements probably did not capture key heated areas. Yet, the fact that similar orders of magnitudes are obtained experimentally and numerically is encouraging.

\subsubsection{Analysis of the interaction}

The results presented above allow to better apprehend the evolution of the blade dynamics during the simulated interaction. The succession of three distinct phases and the understanding of each phase's specificity is assumed to be the cornerstone of the witnessed interaction. The understanding of the transitions from phases 1 to 2 and from phases 2 to 3 greatly benefits from the numerical model since experimental wear patterns at these instants are experimentally inaccessible.

Phase 1: following the very first blade-tip/abradable coating contacts at the leading edge, the abradable coating is worn off (wear levels are very low due to the reduced incursion depth) and only a few microns of abradable coating are removed from a revolution to another. These repeated contacts yield low amplitudes of vibration 
on the blade but do generate a significant amount of energy that heats up the ovalized casing. The casing is thus regularly heated at the two privileged contact locations, in front of the blade's leading edge. Over time, the diffusion of heat within the casing leads to significant thermal expansions around the impacted areas both circumferentially and axially, towards the trailing edge. During this phase, the blade dynamics involves both modes $1 \mathrm{~B}$ and $1 \mathrm{~T}$. The transition between phases 1 and 2 is characterized by the fact that thermal expansions at the trailing edge become sufficient to close the blade-tip/casing clearance and thus initiate contact.

Phase 2: during this phase, contact occurs all along the blade chord, from the leading edge to the trailing edge. This contact configuration is less favorable to torsion motions that are not compatible with simultaneous contacts at the leading edge and the trailing edge. For that reason, bending induced motions are favored during phase 2. Nonetheless, there is a competition between torsion and bending modes all along this phase. As the amplitudes of vibration increase, contacts become more severe: contact forces applied on the blade-tip increase significantly. As the overall energy in the system increases, the eigenfrequencies of the impacting blade also increase due to contact stiffening. It is then assumed that this increase has a twofold effect: (1) increasing the $1 \mathrm{~T}$ eigenfrequency tends to accelerate the desynchronization of torsion induced motions with the blade angular speed. To the contrary, (2) increasing the 1B eigenfrequency synchronizes bending motions with the blade angular speed along the seventh engine order. The transition between phases 2 and 3 is characterized by the perfect synchronization of the blade dynamics with its angular speed.

Phase 3: once the blade vibration is synchronous, it vibrates along its first bending mode along the seventh engine order, this is why seven deep wear lobes are found at the trailing edge. The absence of a seven lobe pattern at the leading edge is simply related to the fact that the blade vibration along its first bending mode yields only very low radial amplitudes of vibration at the leading edge.

In the end, it is remarkable that thermomechanics is key to accurately predict the divergent behavior that was witnessed experimentally in [23]. Nonetheless, the different time scales associated with each interaction phase may provide additional insight on the respective contributions of thermomechanics and dynamics in the arisal of the interaction. The long duration of phase 1 and the key event that is the initiation of contact at the blade trailing edge are undoubtedly related to thermomechanics. However, once the blade dynamics is driven by its first bending mode, during phases 2 and 3, time scales are much shorter and it thus seems probable that the divergent behavior of the blade is only associated to the blade's intrinsic dynamics.

\section{Conclusion}

This contribution focuses on the multi-physics simulation of rubbing events in aircraft engines. An original numerical procedure is introduced that combines an existing in-house numerical strategy for contact and wear management with a newly developed thermomechanical approach relying on the commercial software Ansys. The convergence of the proposed numerical strategy is assessed with respect to its key numerical parameters before it is validated on a rubbing event that was experimentally simulated on a full-scale compressor stage.

The good agreement between the numerical model and experimental observations is underlined in terms of wear profiles and maximum stresses within the blade. New insight is also provided with respect to the experimental set-up and the possible inappropriate location of temperature probes. The in-depth comparison of the numerical results with experimental observations allows to (1) significantly improve the fidelity of numerical predictions thanks to the consideration of thermomechanics on the casing and, (2) to propose a detailed analysis of the contact scenario yielding to divergent amplitudes of vibration.

Presented results allow for a better understanding of the respective role of thermomechanics and dynamics in the arisal of the interaction. In particular, the key role of thermomechanics in the casing distortion that yields a synchronization of the blade dynamics with the seventh engine order is put forward.

\section{Acknowledgment}

This research was supported by Safran Aircraft Engines. The authors are grateful to Antoine Millecamps, former engineer of Safran Aircraft Engineers who initiated and contributed to this research work throughout the years on both experimental and numerical aspects. 


\section{References}

[1] Parent, M.-O., Thouverez, F., and Chevillot, F., 2014. "Whole engine interaction in a bladed rotor-to-stator contact". In Proceedings of the ASME Turbo Expo 2014, GT2014-25253. doi: 10.1115/GT2014-25253 - oai: hal-01223063.

[2] Salvat, N., Batailly, A., and Legrand, M., 2016. "Two-dimensional modeling of unilateral contact-induced shaft precessional motions in bladed-disk/casing systems". International Journal of Non-Linear Mechanics, 78, pp. 90-104. doi: 10.1115/GT2014-25688 - oai: hal-01090671.

[3] Jacquet-Richardet, G., Torkhani, M., Cartraud, P., Thouverez, F., Nouri Baranger, T., Herran, M., Gibert, C., Baguet, S., Almeida, P., and Peletan, L., 2013. "Rotor to stator contacts in turbomachines. Review and application". Mechanical Systems and Signal Processing, 40(2), pp. 401-420. doi: 10.1016/j.ymssp.2013.05.010 - oai: hal-00934050.

[4] Borel, M., Nicoll, A., Schlapfer, H., and Schmid, R., 1989. "The wear mechanisms occurring in abradable seals of gas turbines". Surface \& Coatings Technology, 39, pp. 117-126. doi: 10.1016/0257-8972(89)90046-7 - oai: hal-01553649.

[5] Turner, K. E., Dunn, M., and Padova, C., 2012. "Airfoil Deflection Characteristics During Rub Events". Journal of Turbomachinery, 134(1), p. 011018. doi: 110.1115/1.4003257.

[6] Batailly, A., Legrand, M., Millecamps, A., and Garcin, F., 2015. "Conjectural bifurcation analysis of the contact-induced vibratory response of an aircraft engine blade". In Journal of Sound and Vibration, Vol. 348, Elsevier, pp. 239-262. doi: 10.1016/j.jsv.2015.03.005 - oai: hal-01223575.

[7] Williams, R. J., 2011. "Simulation of Blade Casing Interaction Phenomena in Gas Turbines Resulting from Heavy Tip Rubs Using an Implicit Time Marching Method". In ASME Turbo Expo 2011: Power for Land, Sea and Air, pp. 1-10. doi: 10.1115/GT2011-45495 - oai: hal-01555287.

[8] Legrand, M., Batailly, A., Magnain, B., Cartraud, P., and Pierre, C., 2012. "Full three-dimensional investigation of structural contact interactions in turbomachines". Journal of Sound and Vibration, 331(11), pp. 2578-2601. doi: 10.1016/j.jsv.2012.01.017 - oai: hal-00660863.

[9] Bounazef, M., Guessasma, S., and B., A. S., 2004. "The wear, deterioration and transformation phenomena of abradable coating BN-SiAl-bounding organic element, caused by the friction between the blades and the turbine casing". Materials Letters, 58, pp. 3375-3380. doi: 10.1016/j.matlet.2004.02.049.

[10] Sutter, G., Philippon, S., and Garcin, F., 2006. "Dynamic analysis of the interaction between an abradable material and a titanium alloy". Wear, 261, pp. 686-692. doi: 10.1016/j.wear.2006.01.030 - oai: hal-01223431.

[11] Padova, C., Barton, J., Dunn, M. G., Manwaring, S., Young, G., Adams, M. J., and Adams, M., 2005. "Development of an experimental capability to produce controlled blade tip/shroud rubs at engine speed". Journal of Turbomachinery, 127(4), pp. 726-735. doi: 10.1115/1.1934429 - oai: hal-01553829.

[12] Dadouche, A., Conlon, M. J., Dmochowski, W., Liko, B., and Bedard, J. P., 2008. "Experimental evaluation of abradable seal performance at high temperature". In Proceedings of the ASME Turbo Expo 2008 - GT2008-51228. doi: 10.1115/GT2008-51228 - oai: hal-01430519.

[13] Baiz, S., Fabis, J., Boidin, X., and Desplanques, Y., 2013. "Experimental investigation of the blade/seal interaction". Proceedings of the Institution of Mechanical Engineers, Part J: Journal of Engineering Tribology. doi: 10.1177/1350650112472853 - oai: hal-00863616.

[14] Mandard, R., Witz, J.-F., Boidin, X., Fabis, J., Desplanques, Y., and Meriaux, J., 2015. "Interacting force estimation during blade/seal rubs". Tribology International, 82, pp. 504-513. doi: 10.1016/j.triboint.2014.01.026 - oai: hal-01166811.

[15] Cuny, M., Philippon, S., Chevrier, P., and Garcin, F., 2014. "Experimental measurement of dynamic forces generated during short-duration contacts: application to blade-casing interactions in aircraft engines". Experimental Mechanics, 54, pp. 101-114. doi: 10.1007/s11340-013-9780-z - oai: hal-01430522. 
[16] Delebarre, C., Wagner, V., Paris, J.-Y., Dessein, G., Denape, J., and Santanach, J. G., 2017. "Tribological characterization of a labyrinth-abradable interaction in a turbo engine application". Wear, 370-371, pp. 29-38. doi: 10.1016/j.wear.2016.11.007 - oai: hal-01758599.

[17] Padova, C., Dunn, M. G., Barton, J., Turner, K., Turner, A., and DiTommaso, D., 2011. "Casing treatment and blade-tip configuration effects on controlled gas turbine blade tip/shroud rubs at engine conditions". Journal of Turbomachinery, 133(1), p. 011016. doi: 10.1115/1.4000539.

[18] Ma, X., and Matthews, A., 2007. "Investigation of abradable seal coating performance using scratch testing". Surface 83 Coatings Technology, 202, pp. 1214-1220. doi: 10.1016/j.surfcoat.2007.07.076 - oai: hal-01430526.

[19] Ma, X., and Matthews, A., 2009. "Evaluation of abradable seal coating mechanical properties". Wear, 267(9-10), pp. 1501-1510. doi: 10.1016/j.wear.2009.03.044 - oai: hal-01430513.

[20] Padova, C., Barton, J., Dunn, M., and Manwaring, S., 2007. "Experimental results from controlled blade tip/shroud rubs at engine speed". Journal of Turbomachinery, 129, pp. 713-723. doi: 10.1115/1.1934429 - oai: hal-01553829.

[21] Millecamps, A., 2010. "Interaction aube-carter : contribution de l'usure de l'abradable et de la thermodynamique sur la dynamique d'aubes (Blade-casing interaction : contribution of the abradable wear and the thermomechanical behavior on the blade dynamics)". PhD thesis. oai: tel-01200536.

[22] Batailly, A., Legrand, M., Millecamps, A., and Garcin, F., 2012. "Numerical-experimental comparison in the simulation of rotor/stator interaction through blade-tip/abradable coating contact". Journal of Engineering for Gas Turbines and Power, 134(8), pp. 082504-01-11. doi: 10.1115/1.4006446 - oai: hal-00746632.

[23] Millecamps, A., Brunel, J.-F., Dufrenoy, P., Garcin, F., and Nucci, M., 2009. "Influence of thermal effects during blade-casing contact experiments". In ASME 2009 International Design Engineering Technical Conferences and Computers and Information in Engineering Conference, pp. 855-862. doi: 10.1115/DETC2009-86842 - oai: hal-01223060.

[24] Batailly, A., Legrand, M., and Pierre, C., 2016. "Full 3D Rotor/Stator Interaction Simulations in Aircraft Engines With Time-Dependent Angular Speed". In ASME Turbo Expo 2016: Turbomachinery Technical Conference and Exposition, pp. 1-10. doi: 10.1115/1.4034503 - oai: hal-01388425.

[25] Guérin, N., Thouverez, F., Gibert, C., Legrand, M., and Almeida, P., 2017. "Thermomechanical component mode synthesis for blade casing interaction prediction". In Proceedings of the ASME Turbo Expo 2017 conference, GT2017-64342. doi: 10.1115/GT2017-64342 - oai: hal-01569918.

[26] Nyssen, F., and Batailly, A., 2018. "Thermo-mechanical modeling of abradable coating wear in aircraft engines". Journal of Engineering for Gas Turbines and Power, 141(2), p. 021031. doi: 10.1115/GT2018-75824 - oai: hal-02136818.

[27] Agrapart, Q., 2018. "Bilan d'énergie au contact et contribution de la thermomécanique sur la dynamique d'interaction aube - revêtement abradable de turboréacteur aéronautique (Energy balance and thermomechanical effect on the dynamic interaction between blades and abradable coating in turboengine)". PhD thesis, Université de Lille.

[28] Legrand, M., Batailly, A., and Pierre, C., 2011. "Numerical investigation of abradable coating removal through plastic constitutive law in aircraft engine". Journal of Computational and Nonlinear Dynamics, $\mathbf{7}$, pp. 011010-01-11. doi: 10.1115/1.4004951 - oai: hal-00627526.

[29] Carpenter, N. J., Taylor, R. L., and Katona, M. G., 1991. "Lagrange constraints for transient finite element surface contact". International journal for numerical methods in engineering, 32(1), pp. 103-128. doi: 10.1002/nme.1620320107 - oai: hal-01389918.

[30] Batailly, A., Cuny, M., Legrand, M., and Philippon, S., 2013. "Numerical-experimental confrontation in the simulation of tool/abradable material interaction". Journal of Engineering for Gas Turbines and Power, 135. doi: 10.1115/1.4023262 - oai: hal-00826981. 
[31] Guérin, N., Thorin, A., Thouverez, F., Legrand, M., and Almeida, P., 2018. "Thermomechanical model reduction for efficient simulations of rotor-stator contact interaction". p. 022501. doi: 10.1115/GT2018-75880 - oai: hal-01713849.

[32] Thorin, A., Guérin, N., Legrand, M., Thouverez, F., and Almeida, P., 2018. "Nonsmooth thermoelastic simulations of blade-casing contact interactions". p. 022502. doi: 10.1115/1.4040857 - oai: hal-01989188.

[33] Laverty, W., 1982. "Rub energetics of compressor blade tip seals". Wear, 75(1), pp. 1-20. doi: 10.1016/00431648(82)90137-5 .

[34] Laraqi, N., Alilat, N., de Maria, J. G., and Baïri, A., 2009. "Temperature and division of heat in a pin-on-disc frictional device exact analytical solution". Wear, 266(7-8), pp. 765-770. doi: 10.1016/j.wear.2008.08.016.

[35] Yi, M.-Z., Zhang, X.-L., Hu, N.-S., and He, J.-W., 1999. "Abradability evaluation of seal coating by impactscraping test machine". Acta Aeronautica et Astronautica Sinica, 20(3), pp. 249-253.

[36] Craig, R., and Bampton, M., 1968. "Coupling of substructures for dynamic analyses". AIAA Journal, 6(7), pp. 1313-1319. doi: 10.2514/3.4741 - oai: hal-01537654. 